\title{
Energy-Efficient Time Synchronization in Wireless Sensor Networks via Temperature-Aware Compensation
}

\author{
MIAO XU, University of South Carolina \\ WENYUAN XU, University of South Carolina, Zhejiang University \\ TINGRUI HAN and ZHIYUN LIN, Zhejiang University
}

\begin{abstract}
Time synchronization is critical for wireless sensor networks (WSNs) because data fusion and duty cycling schemes all rely on synchronized schedules. Traditional synchronization protocols assume that wireless channels are available around the clock. However, this assumption is not true for WSNs deployed in intertidal zones. In this article, we present TACO, a synchronization scheme for WSNs with intermittent wireless channels and volatile environmental temperatures. TACO estimates the correlation of clock skews and temperatures by solving a constrained least squares problem and continuously adjusts the local time with the predicted clock skews according to temperatures. Our experiment conducted in an intertidal zone shows that TACO can greatly reduce the clock drift and prolong the resynchronization intervals.
\end{abstract}

Categories and Subject Descriptors: C.2.0 [Computer-Communication Networks]: General

General Terms: Algorithms, Design, Experimentation, Performance

Additional Key Words and Phrases: Wireless sensor networks, temperature aware, time synchronization

ACM Reference Format:

Miao Xu, Wenyuan Xu, Tingrui Han, and Zhiyun Lin. 2016. Energy-efficient time synchronization in wireless sensor networks via temperature-aware compensation. ACM Trans. Sen. Netw. 12, 2, Article 12 (April 2016), 29 pages.

DOI: http://dx.doi.org/10.1145/2876508

\section{INTRODUCTION}

Time synchronization is critical for wireless sensor networks (WSNs) because numerous WSN applications and protocols rely on it. For example, for power efficiency, sensor nodes typically alternate between the sleep and wake-up state [Szewczyk et al. 2004; Tolle et al. 2005; Hartung et al. 2006], and neighboring nodes have to coordinate their wake-up intervals for data transmission, and distributed event monitoring requires synchronization among sensor nodes for correct data fusion. Most existing work [Elson et al. 2002; Ganeriwal et al. 2003; Ping 2003] involves broadcasting messages to synchronize sensor nodes with a reference node, and periodical resynchronization is needed to compensate for the clock drift over time (i.e., the cumulating difference of time between different clocks). For example, resynchronization messages must be broadcast

This work was supported in part by the National Science Foundation under grants CNS-0845671 and GEO1124657, and the National Natural Science Foundation of China under grants 61472358 and 61273113 . The research was done at the University of South Carolina.

Authors' addresses: M. Xu, currently affiliated with Cisco Systems, 3550 Cisco Way, San Jose, CA 95134, USA; email: xum@email.sc.edu; W. Xu (corresponding author), T. Han, and Z. Lin, College of Electrical Engineering, Zhejiang University, 38 Zheda Road, Hangzhou, Zhejiang 310027, China; emails: \{xuwenyuan, hantingrui, linz\}@zju.edu.cn.

Permission to make digital or hard copies of part or all of this work for personal or classroom use is granted without fee provided that copies are not made or distributed for profit or commercial advantage and that copies show this notice on the first page or initial screen of a display along with the full citation. Copyrights for components of this work owned by others than ACM must be honored. Abstracting with credit is permitted. To copy otherwise, to republish, to post on servers, to redistribute to lists, or to use any component of this work in other works requires prior specific permission and/or a fee. Permissions may be requested from Publications Dept., ACM, Inc., 2 Penn Plaza, Suite 701, New York, NY 10121-0701 USA, fax +1 (212) 869-0481, or permissions@acm.org.

(c) 2016 ACM 1550-4859/2016/04-ART12 $\$ 15.00$

DOI: http://dx.doi.org/10.1145/2876508 


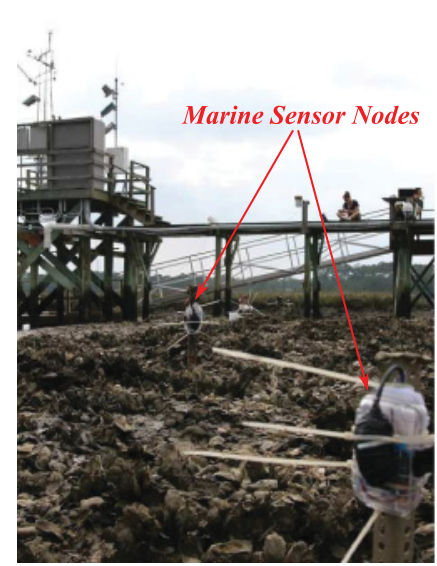

(a) Low tide

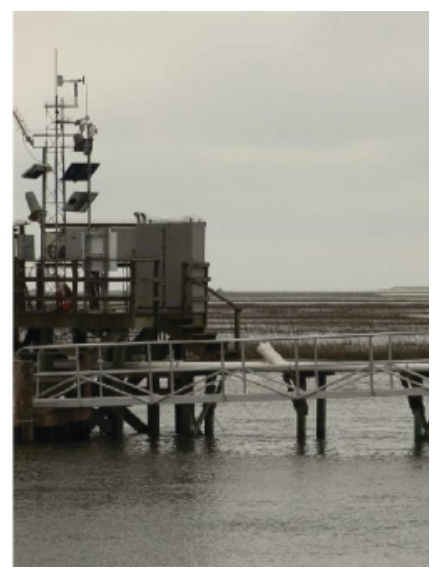

(b) High tide

Fig. 1. An illustration of intermittent wireless channels. (a) Sensor nodes are exposed in the air and can communicate during a low tide. (b) Sensor nodes are submerged underwater and cannot communicate during a high tide.

once every 34 minutes to ensure a time difference less than $10 \mathrm{~ms}$ between neighboring nodes using TPSN [Ganeriwal et al. 2003]. Such synchronization protocols are effective when a wireless communication channel is always available. However, wireless channels could be intermittent due to environmental disturbances. How to maintain time synchronization despite intermittent wireless channels has not been well studied.

In this work, we focus on WSNs that are deployed in intertidal zones (WSN-IT hereafter). The wireless channel in a WSN-IT can become unavailable due to tidal cycles (as shown in Figure 1): submerged by seawater in a high tide, the communication range of sensor nodes reduces to a few centimeters because of the strong signal attenuation in seawater [Vasilescu et al. 2005]. Depending on the tidal level and the altitude, sensor nodes can remain underwater up to 24 hours, making the aforementioned messagebased synchronization protocols ineffective. Unsynchronized clocks are problematic, especially right after sensors are exposed to the air during low tides-sensors just regain their communication ability and want to upload the buffered measurements, but their clocks are unsynchronized and unable to coordinate data upload among neighbors. A time synchronization protocol that can estimate the clock drift without relying on channel availability is needed.

In addition, deployed outdoors, sensor nodes are subject to volatile temperature changes, and they may experience different temperatures depending on their installation locations (e.g., in/out of a shadow, below/above sea level). For instance, Figure 2 shows the temperatures measured by two nodes on a hot summer day: one node was placed on dry land that was not affected by tidal cycles, and the other was in an intertidal zone that was periodically submerged by seawater. Both nodes underwent a large temperature change ranging from $23^{\circ} \mathrm{C}$ to $50^{\circ} \mathrm{C}$, but each observed different temperatures over time. Affected by temperatures, the clock skews (i.e., the instantaneous variance of the clock drift rate) of the two nodes diverged. Most existing methods for clock skew estimation do not consider temperature and are insufficient for synchronization in WSN-IT.

In this article, we design a synchronization protocol utilizing temperature-aware compensation (TACO) for WSN-IT where the wireless channel is intermittent and sensor nodes may experience volatile and distinct temperature changes. Essentially, TACO is motivated by the observation that clock skews and temperature variations are highly correlated and in a quadratic relationship. Thus, during the initialization phase, sensor 


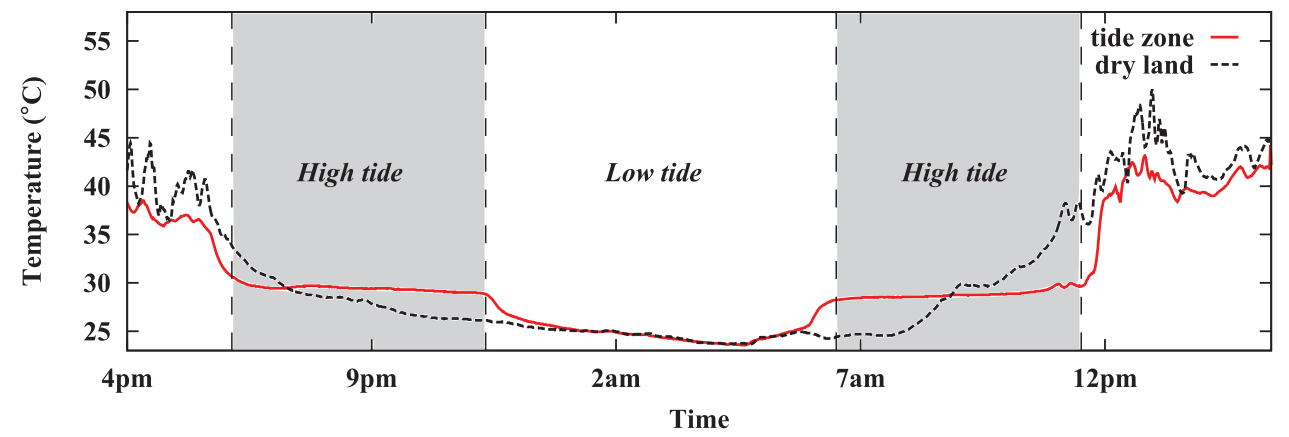

Fig. 2. Temperatures recorded over 24 hours in Georgetown, South Carolina. One node was mounted in an intertidal zone and the other on dry land. Their time drift was $7.5 \mathrm{~ms}$ and $13 \mathrm{~ms}$ after the first and second high tide periods, respectively.

nodes can estimate the quadratic relationship with synchronization messages. Thereafter, they continuously adjust their local time according to the environmental temperature. TACO can be used together with broadcast-based synchronization schemes to prolong the resynchronization interval; thus, in this sense, it is complementary to many existing synchronization protocols [Elson et al. 2002; Ganeriwal et al. 2003; Ping 2003].

Prior work [Schmid et al. 2009; Yang et al. 2012] has exploited temperature for estimating clock skew but relies on a mapping table from temperatures to clock skews. To adjust time, a sensor node looks up the table based on its current temperature. If the current temperature is out of the temperature range in the table, a resynchronization is triggered to estimate the clock skew for that temperature. Such a scheme is inapplicable to WSN-IT, where the wireless channel could be unavailable for up to 24 hours. In comparison, TACO models the correlation between clock skews and temperature variations. With a few measurements, a sensor node can estimate the correlation efficiently and predict the clock skews for unseen temperatures, without resynchronization broadcast. The contributions of the article include the following:

-We propose to predict clock skew based on its correlation to temperature and to estimate the correlation mathematically using a least squares method.

-We implement TACO, a time synchronization protocol that estimates the correlation between temperatures and clock skews, and adjusts the clock based on environmental temperatures.

-We present an energy model to describe the relationship between energy consumption and time synchronization. The model considers various energy costs, including computations, temperature sensing, and communications.

We organize the rest of the article as follows. We introduce our network model and overview the problem in Section 2. In Section 3, we discuss the clock skew basics and formulate the correlation. We present methods to measure clock skews in Section 4 and implementation details in Section 5. In Section 6, we evaluate the performance of TACO through in-field tests. In Section 7, we formulate an energy model to analyze the energy efficiency of TACO. We discuss the related work in Section 8 and conclude our work in Section 9.

\section{PROBLEM OVERVIEW}

In this section, we introduce the WSNs for intertidal zones (WSN-IT) and overview our time synchronization scheme. 


\subsection{WSN-IT Architecture}

Similar to prior WSNs [Tolle et al. 2005; Hartung et al. 2006], a WSN-IT also consists of two types of nodes: a sink node and a collection of marine sensor nodes.

Marine sensor nodes are battery powered and deployed in intertidal zones. They are subject to tidal cycles and may be submerged by seawater periodically. Each node has on-board sensors to monitor the environment parameters (e.g., temperatures) and a radio transceiver for communication. Because sensor nodes are battery powered, they use a duty cycling scheme to reduce energy consumption.

A sink node collects the measurements from marine sensor nodes and relays data to a data center. Unlike marine sensor nodes, the sink node is kept on dry land and is unaffected by tidal cycles. Without loss of generality, we assume that the sink node serves as a reference node ${ }^{1}$ that provides the reference time to marine sensor nodes. Equipped with advanced hardware, we assume that the sink node does not suffer from any clock skews caused by temperature variance. Later we will lift this assumption and address the issue if the sink node also suffers from clock skews.

\subsection{WSN-IT Features}

Figure 1 shows one of our deployment sites. During a low tide period, marine sensor nodes are out of water, and during a high tide period, the nodes are submerged by seawater. This distinct environment change makes our sensor network exhibit the following two important characteristics.

Intermittent wireless channels. When nodes are underwater, their wireless channel is unavailable due to the large signal attenuation, which can be calculated by $\alpha=$ $0.0173 \times \sqrt{f \times \sigma}$, where $f$ is the radio frequency in Hertz and $\sigma$ is the conductivity of the seawater (typically $4 \mathrm{mhos} / \mathrm{m}$ [Butler 1987]). Consider a sensor network operating at $916 \mathrm{MHz}$, the attenuation underwater is $1,046 \mathrm{~dB} / \mathrm{m}$, which results in a communication range of less than $12 \mathrm{~cm}$ at a transmission power of $10 \mathrm{dBm}$.

Thus, nodes are unable to communicate with each other during a high tide period. Depending on the tidal level and the installation location, the channel of a sensor node could remain unavailable for up to 24 hours. Intermittent wireless channels demand new synchronization strategies for WSN-IT networks.

Large temperature variations. Similar to many outdoor sensor networks, a WSNIT is subject to large temperature changes caused by weather conditions and sun exposure. For example, a node that is under the shadow of a rock will experience a lower temperature than the one that is directly exposed to sunlight. In addition, the temperatures of marine sensor nodes are affected by tidal cycles. For instance, on a hot summer day, when the tide comes in, the node's temperature drops quickly to the seawater temperature even if the air temperature is high. Figure 2 illustrates such a scenario-the temperatures of the node in the intertidal zone was relatively stable compared to the node on dry land.

To understand the impact of temperatures on clock skews, we exposed a marine sensor node to various temperatures and measured the clock drifts accumulated over 24 hours. The results (depicted in Figure 3) show that temperature is a nonnegligible factor with respect to clock skews-the node's clock became 1.24s slower than the reference time after 24 hours if exposed to $43^{\circ} \mathrm{C}$, and became $0.06 \mathrm{~s}$ slower if exposed to $26^{\circ} \mathrm{C}$. Recall the example illustrated in Figure 2. Exposed to different temperatures, two marine sensor nodes' clocks drifted $7.5 \mathrm{~ms}$ and $13 \mathrm{~ms}$ apart at the end of the first and second high tide cycles, respectively, even if their clocks were synchronized at the beginning of each high tide cycle.

\footnotetext{
${ }_{1}^{1}$ The terms reference node and sink node are used interchangeably in this article.
} 


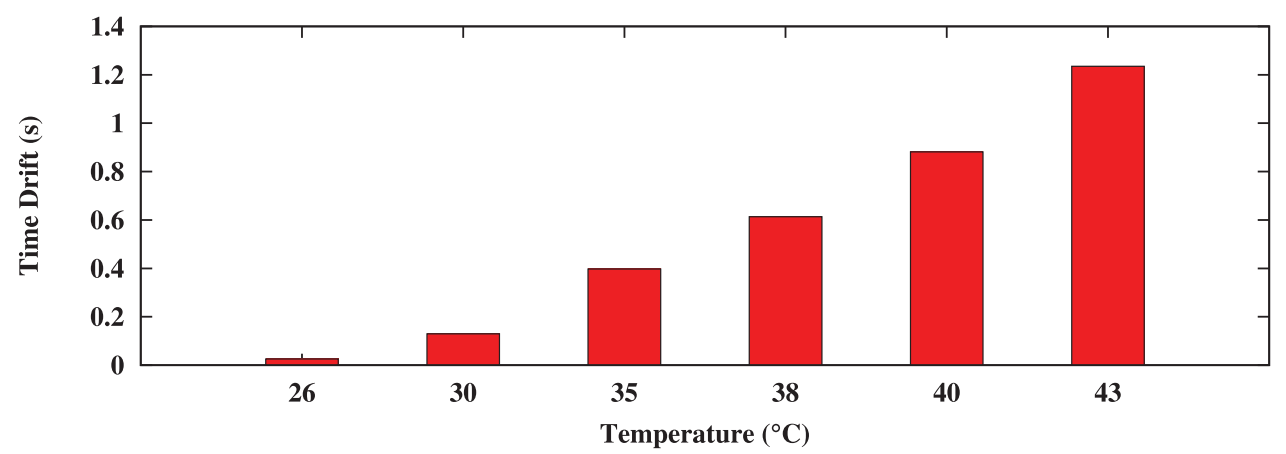

Fig. 3. The time drifts of a marine sensor node accumulated in 24 hours. The drift increases with an increase in temperature.

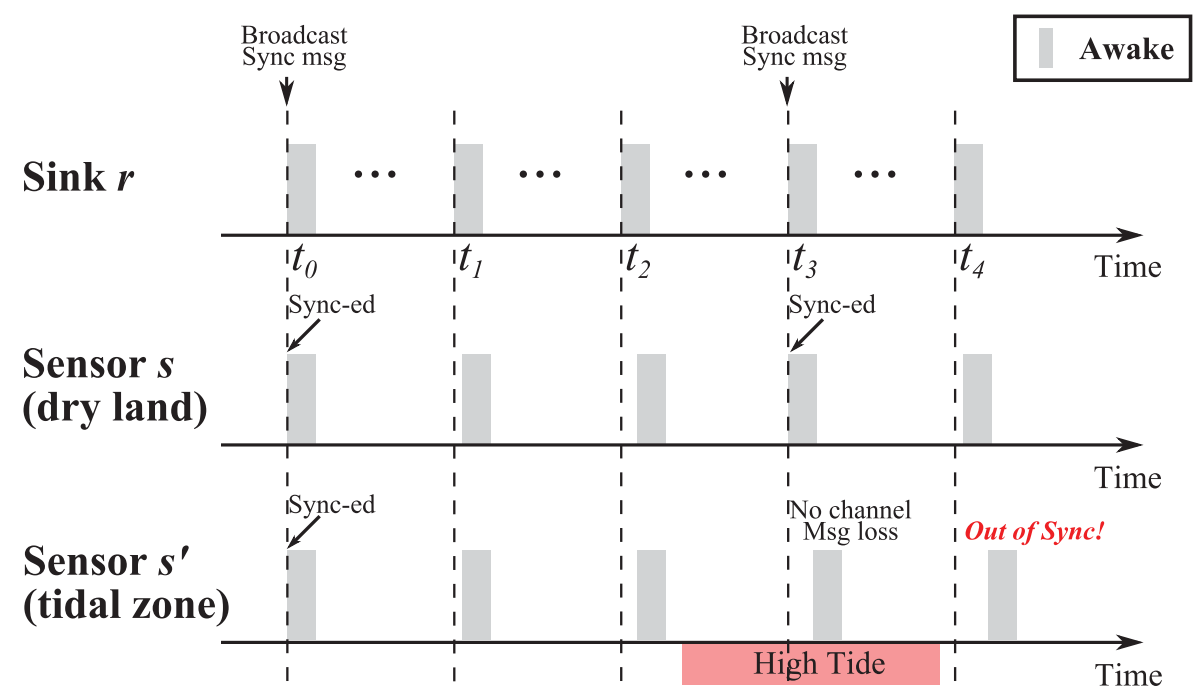

Fig. 4. Illustration of failed broadcast-based synchronization. Node $s$ is synchronized with sink $r$ at $t_{3}$, whereas node $s^{\prime}$ is out of synchronization after $t_{4}$ due to missing synchronization messages at $t_{3}$.

The large temperature range and temperature variance between outdoor nodes result in bigger clock drift than in indoor WSNs, making time synchronization in WSN-IT challenging.

\subsection{TACO Overview}

Since the WSN-IT alternates between the sleep and wake-up states, the synchronized time is critical for scheduling nodes' wake-up periods. Figure 4 illustrates the scenarios where traditional broadcast-based synchronization schemes fail: sensors $s$ and $s^{\prime}$, which are deployed on dry land and an intertidal zone, respectively, try to synchronize their clocks with the reference node $\left(\operatorname{sink} r\right.$ ). At $t_{0}$, both sensors are synchronized. As time elapses, their wake-up periods lag behind the $r$ 's due to the clock drift. At time $t_{3}$, a resynchronization message from $r$ enables the sensor $s$ to update its clock for aligning its wake-up periods with $r$ 's. However, the sensor $s^{\prime}$, submerged by seawater in a high tide period, is unable to receive resynchronization messages and will eventually result in a completely misaligned wake-up period. 
A naive solution would be to increase the wake-up duration, although at the cost of excessive energy consumption on idle listening. In this work, we propose a time synchronization scheme (TACO) that lets sensors predict the clock drift and adjust their clock locally without frequent resynchronization. Particularly, TACO exploits the correlation of clock skews and temperatures, which can be formulated by a quadratic function. Once the correlation is identified, sensor nodes can estimate the clock skews and perform time compensation based on the environmental temperatures.

TACO consists of two stages: the initialization stage and the compensation stage. The initialization stage typically happens during a low tide cycle with stable wireless channels. All sensors form a tree-based network topology that is rooted at the sink. The sink node broadcasts timestamped beacons periodically. Each sensor node estimates its clock skews with the help of beacons and measures temperatures. Once enough clock skew and temperature pairs are collected, the sensor node estimates the correlation. In the compensation stage, sensor nodes measure temperatures, calculate clock skews based on the correlation estimated during initialization, and adjust local clocks.

TACO is ideal for WSN-IT networks because it only requires reliable wireless communication during initialization and adjusts the clock locally without constant communication. However, there are a few challenges associated with this compensation scheme:

(1) Are temperatures the dominate factor affecting clock skews? How do we quantify the correlation of temperatures and clock skews?

(2) How do we measure the clock skews?

(3) How do we adjust the local time given that the correlation is known?

We address all of the preceding questions in the following sections.

\subsection{Definitions and Notation}

We follow the convention of common terms [Sundararaman et al. 2005] related to clocks, which are defined next.

Clock is a device that ticks at a certain rate and counts the number of ticks from a starting point.

Time is the number of ticks reported by a clock. Denote $C^{s}(t)$ as the local time of a sensor node $s$ at the reference time $t$. The time of the reference node $r$ is $C^{r}(t)=t$.

Clock offset is the difference between the local time and the reference time. The clock offset of a sensor node $s$ at $t$ is $O^{s}(t)=C^{s}(t)-t$.

Clock frequency is the rate at which a clock ticks (i.e., the first derivative of the time). Let $C^{s}(t)$ be the time of a node $s$, then $\dot{C}^{s}(t)$ is the clock frequency at $t$. The clock frequency of the reference node $r$ is 1 .

Clock skew is the difference of clock frequency between a node $s$ and the reference node $r$. Let $C^{s}(t)$ be the time of the node $s$; the clock skew at time $t$ is $P^{s}(t)=\dot{C}^{s}(t)-t=$ $\dot{O}^{s}(t)$.

Typically, the clock accuracy is described by the clock skew and measured in parts per million (ppm). For example, a clock skew of $20 \mathrm{ppm}$ will lead to a clock drift of $1.73 \mathrm{~s}$ perday from the reference time.

\section{CLOCK SKEW MODELING}

In this section, we examine the correlation of clock skews and temperatures. We first introduce crystal oscillators and the sources of clock skews, and then model the correlation as a constrained least squares problem. 


\subsection{Crystal Basics}

3.1.1. Crystal Oscillators. A crystal oscillator is an electronic circuit that vibrates continuously to create electrical signals. It is typically used to keep track of time, and quartz crystals are typically utilized. For example, the popular Telos [Polastre et al. 2005] motes use a quartz crystal oscillating at $32.768 \mathrm{kHz}$ to maintain the real-time clock (RTC).

Crystal oscillators can be cut into different shapes, such as a rectangular plate or a tuning fork. For time keeping, the most widely used is the tuning fork because of its smaller size, lower price, and higher accuracy [Lam 2008]. Thus, in this article, we focus on tuning fork cut crystals.

3.1.2. Sources of Clock Skews. The clock accuracy depends on the accuracy of a crystal's vibration frequency. Any vibration deviation of the crystal will result in a clock drifting away from the reference time. The deviations are mainly caused by mechanical vibration interference, mismatched load capacitance, environmental temperature changes, and crystal aging [Microchip 2008; Walls and Gagnepain 1992].

Mechanical vibration sources could be speakers, buzzers, motors, and the like [Microchip 2008]. To avoid the interference of mechanical vibration, potential vibration sources should be removed from the vicinity of crystals when deploying sensor nodes or designing the sensor circuit.

Another source of clock skews is mismatched load capacitors, which are used to ground a crystal. A mismatched capacitor could lead to significant crystal errors [Walls and Gagnepain 1992]. To avoid this problem, the capacitors recommended by the crystal's specifications should be chosen.

Environmental temperature is another major source that causes clock skews. The vibration frequency of the crystal changes as the temperature varies [Walls and Gagnepain 1992]. Because sensor networks are usually deployed outdoors and subject to temperature change, the temperature effect cannot be neglected. To overcome this problem, the temperature-compensated crystal oscillator (TCXO) could be used. However, TCXO increases the cost and the power consumption [Schmid et al. 2009], and thus it is not suitable for sensor nodes due to limited resources.

All crystals are subject to aging, which is defined as "a systematic change in frequency with time due to internal changes in the oscillator" [Lombardi 2001]. Aging is usually not observed until a crystal oscillator operates after a certain period (e.g., within the first 2 months through the first year). This crystal aging will lead to frequency change. Taking the crystal oscillators in marine sensor nodes as an example, the frequency change caused by aging is up to $3 \mathrm{ppm}$ per year, which is as much as $15 \%$ of the frequency tolerance [Epson 2014]. The frequency tolerance is defined as the initial deviation of a crystal oscillator frequency as compared to the absolute frequency at room temperature.

In summary, some sources of clock skews, such as vibration interference and mismatched capacitors, could be avoided by designing and deploying the sensor nodes carefully. However, the error due to temperature changes and crystal aging presents a challenge to system designers because they are unknown during the design process and hence must be taken care of during its runtime execution [Microchip 2008]. Motivated by this observation, we propose TACO, which focuses on estimating clock skews caused by dynamic environment conditions.

\subsection{Temperature Versus Clock Skews}

Like many other types of crystal oscillators, tuning fork cut crystals are sensitive to temperature variations. This characteristic is described by temperature coefficient (denoted by $\alpha_{T}$ ), which is defined as the ratio of clock skew difference over the temperature 
difference to the turnover temperature ${ }^{2}$ (denoted by $T_{0}$ ) - that is, $P_{T}-P_{0}=\alpha_{T}\left(T-T_{0}\right)$, where $P_{T}$ and $P_{0}$ are the clock skews at the temperature $T$ and $T_{0}$, respectively. Research has shown that for quartz crystal oscillators, $\alpha_{T}$ is a linear function of the temperature [Strout 1928] (i.e., $\alpha_{T}=k\left(T-T_{0}\right)$ ). Here, $k$ is a constant depending on a crystal's physical property. Thus, the correlation of the clock skew and the temperature can be formulated as a quadratic function:

$$
P_{T}=P_{0}+k \times\left(T-T_{0}\right)^{2},
$$

where $k, T_{0}$, and $P_{0}$ are constants determined by a crystal's physical properties.

Tuning fork crystal oscillators are typically cut in such a way that $T_{0}$ is within a temperature range and $k$ is a negative value. For instance, a typical range of $T_{0}$ is $25^{\circ} \mathrm{C} \pm 5^{\circ} \mathrm{C}$, and the typical value of $k$ is around $-0.04 \mathrm{ppm} /{ }^{\circ} \mathrm{C} ;{ }^{2}$. Formally, $k$ and $T_{0}$ have the following constraints:

$$
\left\{\begin{array}{l}
k<0 \\
\Gamma_{1} \leq T_{0} \leq \Gamma_{2}
\end{array}\right.
$$

where $\Gamma_{1}$ and $\Gamma_{2}$ are the lower bound and upper bound of $T_{0}$.

\subsection{Problem Formulation}

As modeled by Equation (1), the key to estimate the correlation of clock skews and temperatures is to obtain the constants $k, T_{0}$, and $P_{0}$. The basic idea of estimating the constants is to measure a set of clock skews $P_{i}$ at different temperatures $T_{i}$ and then form and solve a set of equations. Suppose that we have $N$ observations of clock skews at different temperatures (i.e., $\left.\Omega=\left\{\left(P_{i}, T_{i}\right) \mid i \in[1, N], N \geq 3\right\}\right)$. Then we have $N$ equations:

$$
P_{i}=P_{0}+k \times\left(T_{i}-T_{0}\right)^{2} .
$$

Expanding these equations, we obtain

$$
P_{i}=k T_{i}^{2}-2 k T_{0} T_{i}+\left(P_{0}+k T_{0}^{2}\right) .
$$

To simplify the explanation, we define

$$
\left(\beta_{1}, \beta_{2}, \beta_{3}\right)=\left(k,-2 k T_{0}, P_{0}+k T_{0}^{2}\right),
$$

and we obtain the following linear model:

$$
y=X \beta,
$$

where

$$
y^{t}=\left(P_{1}, \ldots, P_{N}\right), \beta^{t}=\left(\beta_{1}, \beta_{2}, \beta_{3}\right),
$$

and

$$
X=\left[\begin{array}{ccc}
T_{1}^{2} & T_{1} & 1 \\
\vdots & \vdots & \vdots \\
T_{N}^{2} & T_{N} & 1
\end{array}\right]
$$

Given that $N \gg 3$, the linear model is overdetermined. Therefore, we can solve the problem using the least squares method. Let $\|\cdot\|_{2}$ be the Euclidean norm and $\|\cdot\|_{F}$ be the Frobenius norm; we define the problem of estimating the correlation as a constrained least squares (LSQ) problem as follows.

\footnotetext{
${ }^{2}$ Turnover temperature refers to the temperature when $\alpha_{T}=0$.
} 
Problem 1.

$$
\begin{array}{ll}
\min _{\beta} & \|y-X \beta\|_{2}^{2} \\
\text { s.t. } & A \beta \leq b
\end{array}
$$

where

$$
A=\left[\begin{array}{ccc}
-2 \Gamma_{1} & -1 & 0 \\
2 \Gamma_{2} & 1 & 0 \\
1 & 0 & 0
\end{array}\right], \text { and } b=\left[\begin{array}{c}
0 \\
0 \\
-\epsilon
\end{array}\right]
$$

In particular, $\Gamma_{1}$ and $\Gamma_{2}$ are constant values mentioned in Equation $(2 \mathrm{~b})$ and $\epsilon$ is a positive constant that is sufficiently small to ensure Equation (2a).

We stress that it is important to include the constraints, because clock skew measurements contain errors and the constraints can limit the estimation errors.

To tackle this problem, we employ the active-set dual method that was proposed by Goldfarb and Idnani [1983]. This dual algorithm starts with an unconstrained objective function and then iterates based on updating the result of Cholesky factorization and QR factorization until primal feasibility is available. Compared to some other methods [Fletcher 1971; Goldfarb 1972], this algorithm achieves better performance in terms of time complexity. For more details, we refer the reader to Goldfarb's work [Goldfarb and Idnani 1983]. Once $\beta$ is estimated, we can calculate $k, T_{0}$, and $P_{0}$ as follows:

$$
\left(k, T_{0}, P_{0}\right)=\left(\beta_{1},-\frac{\beta_{2}}{2 \beta_{1}}, \beta_{3}-\frac{\beta_{2}^{2}}{4 \beta_{1}}\right) .
$$

\subsection{Error Analysis}

The performance of TACO is determined by the estimation accuracy of $\beta$. Intuitively, the higher the accuracy of $\beta$, the better the performance of TACO. The accuracy $\beta$ relies on the accuracy of a training set $\Omega=\left\{\left(P_{i}, T_{i}\right)\right\}$. In practice, measurement errors always exist in the training sample $T_{i}$ and $P_{i}$. Nevertheless, one of the advantages of TACO is that the estimation error of $\beta$ is tightly bounded as long as the measurement errors of $T_{i}$ and $P_{i}$ are bounded. In this section, we quantify this bound lies between the estimation error of $\beta$ and the measurement errors of $T_{i}$ and $P_{i}$.

Given the constrained LSQ problem (see Problem (1)), let $X$ and $y$ be the real values and let $\tilde{X}$ and $\tilde{y}$ be the measurement values. They follow

$$
X=\tilde{X}+\Delta X, \text { and } y=\tilde{y}+\Delta y,
$$

where $\Delta X$ and $\Delta y$ are the errors between the measurement values and the real values. Let $\beta$ and $\tilde{\beta}$ be the solutions of the two LSQ problems, respectively-that is, $\beta$ solves the LSQ with the real values $(X, y)$ and $\tilde{\beta}$ solves the problem with the measurement values $(\tilde{X}, \tilde{y})$. Without loss of generality, we assume that $\operatorname{rank}(X)=3$ and $\operatorname{rank}(\tilde{X})=3$ because $N \gg 3$ and all data are collected at different temperatures over a period of time.

We introduce the pseudoinverse of a matrix $X$ here. A pseudoinverse of $X$ is defined as a matrix $X^{\dagger}$ that satisfies the following four criteria:

$$
\begin{aligned}
& X X^{\dagger} X=X, X^{\dagger} X X^{\dagger}=X^{\dagger}, \\
& \left(X X^{\dagger}\right)^{t}=X X^{\dagger},\left(X^{\dagger} X\right)^{t}=X^{\dagger} X
\end{aligned}
$$

Now we show the fact: given that the measurement errors $\Delta X$ and $\Delta y$ are bounded, the estimation error $\Delta \beta$ is bounded as well, where $\Delta \beta=\beta-\tilde{\beta}$. 
THEOREM 3.1. If the measurement errors of clock skews $P_{j}$ and temperatures $T_{j}$ are bounded by $\psi_{1}$ and $\psi_{2}$ for each measurement $j$ :

$$
\left|\tilde{P}_{j}-P_{j}\right| \leq \psi_{1} \text { and }\left|\tilde{T}_{j}-T_{j}\right| \leq \psi_{2},
$$

then

$$
\|\Delta \beta\|_{2} \leq \max \left\{\Sigma_{1}, \Sigma_{2}\right\}
$$

where

$$
\begin{aligned}
\Sigma_{1}= & \max _{i \in[1,6]}\left\{\|\tilde{M}\|_{2}\left(\left\|\Delta b^{i}\right\|_{2}+\left\|\Delta A^{i}\right\|_{2}\|\tilde{\beta}\|_{2}\right)\right. \\
& \left.+\epsilon\left[\left\|(\tilde{X} \tilde{Q})^{\dagger}\right\|_{2}\left(\|\tilde{y}\|_{2}+\|\tilde{X}\|_{F}\|\tilde{\beta}\|_{2}\right)\right]+O\left(\epsilon^{2}\right)\right\},
\end{aligned}
$$

with

$$
\tilde{Q}=I-\left(\tilde{A}^{i}\right)^{\dagger} \tilde{A}^{i}, \tilde{M}=\left(I-(\tilde{X} \tilde{Q})^{\dagger} \tilde{X}\right)\left(\tilde{A}^{i}\right)^{\dagger}
$$

and

$$
\Sigma_{2}=\epsilon\left\|\tilde{X}^{\dagger}\right\|_{2}\left(\|\tilde{y}\|_{2}+\|\tilde{X}\|_{F}\|\tilde{\beta}\|_{2}\right)+O\left(\epsilon^{2}\right) .
$$

In particular, $\epsilon$ is a function of $\psi_{1}$ and $\psi_{2}$, and it tends to 0 when $\psi_{1}$ and $\psi_{2}$ tend to 0 . Moreover, $\Delta A^{i}=-\tilde{A}^{i}$ and $\left[A^{i} \mid b^{i}\right]$ is taken from the following six cases:

$$
\begin{aligned}
& {\left[\begin{array}{ccc|c}
-2 \Gamma_{1} & -1 & 0 & 0
\end{array}\right],\left[\begin{array}{lll|l}
2 \Gamma_{2} & 1 & 0 & 0
\end{array}\right],} \\
& {\left[\begin{array}{ccc|c}
1 & 0 & 0 & -\varepsilon
\end{array}\right],\left[\begin{array}{ccc|c}
-2 \Gamma_{1} & -1 & 0 & 0 \\
2 \Gamma_{2} & 1 & 0 & 0
\end{array}\right],} \\
& {\left[\begin{array}{ccc|c}
-2 \Gamma_{1} & -1 & 0 & 0 \\
1 & 0 & 0 & -\varepsilon
\end{array}\right],\left[\begin{array}{ccc|c}
2 \Gamma_{2} & 1 & 0 & 0 \\
1 & 0 & 0 & -\varepsilon
\end{array}\right] .}
\end{aligned}
$$

PROOF. We start our proof by considering the LSQ problem without constraints-that is,

$$
\min _{\beta}\|y-X \beta\|_{2}^{2}
$$

Because $\operatorname{rank}(X)=3$, which means that $X$ is a full column rank matrix, the solution to Equation (6) is $\beta=X^{\dagger} y$. It falls into two categories:

(1) $\beta$ dose not satisfy $A \beta \leq b$;

(2) $\beta$ satisfies $A \beta \leq b$.

In the first case where $\beta=X^{\dagger} y$ does not satisfy $A \beta \leq b$, the solution to Problem (1) and the solution to Equation (6) are not the same because one or more equality constraints in $A \beta \leq b$ will be active. We use $A^{i} \beta=b^{i}$ to denote the active equalities. Then Problem (1) is changed to

$$
\begin{gathered}
\min _{\beta}\|y-X \beta\|, \\
\text { s.t. } A^{i} \beta=b^{i} .
\end{gathered}
$$

Let $X, y, A^{i}$, and $b^{i}$ present the real value, whereas $\tilde{X}, \tilde{y}, \tilde{A}^{i}$, and $\tilde{b}^{i}$ present the perturbed value. The perturbation norms are measured by the smallest $\epsilon$,

$$
\|\Delta X\|_{F} \leq \epsilon\|\tilde{X}\|_{F},\|\Delta y\|_{2} \leq \epsilon\|\tilde{y}\|_{2},
$$


where

$$
X=\tilde{X}+\Delta X, y=\tilde{y}+\Delta y .
$$

In addition, $A^{i}=\tilde{A}^{i}+\Delta A^{i}, b^{i}=\tilde{b}^{i}+\Delta b^{i}$. And we know that $A^{i}=0$ and $b^{i}=0$ since if there is no measuring error, no equality will be active.

Then we show that three equality constraints cannot be satisfied at the same time by contradiction. Let

$$
A_{1}=\left[\begin{array}{c}
-2 \Gamma_{1} \\
-1 \\
0
\end{array}\right]^{t}, A_{2}=\left[\begin{array}{c}
2 \Gamma_{2} \\
1 \\
0
\end{array}\right]^{t}, A_{3}=\left[\begin{array}{l}
1 \\
0 \\
0
\end{array}\right]^{t}
$$

Suppose that $A_{1} \beta=0, A_{2} \beta=0$, and $A_{3} \beta=-\varepsilon$, then

$$
\left\{\begin{array}{l}
A_{1} \beta=0 \\
A_{2} \beta=0
\end{array} \Rightarrow \beta_{1}\left(\Gamma_{1}-\Gamma_{2}\right)=0 .\right.
$$

Due to the fact that $\beta_{1}=-\varepsilon<0$, we derive $\Gamma_{1}=\Gamma_{2}$. In general, $\Gamma_{1} \neq \Gamma_{2}$, a contradiction. Thus, at most two equalities are active. That is to say, $\left[\tilde{A}^{i} \mid \tilde{b}^{i}\right]$ is taken from the following six cases:

$$
\begin{aligned}
& {\left[\begin{array}{ccc|c}
-2 \Gamma_{1} & -1 & 0 & 0
\end{array}\right],\left[\begin{array}{ccc|c}
2 \Gamma_{2} & 1 & 0 & 0
\end{array}\right],} \\
& {\left[\begin{array}{ccc|c}
1 & 0 & 0 & -\varepsilon
\end{array}\right],\left[\begin{array}{ccc|c}
-2 \Gamma_{1} & -1 & 0 & 0 \\
2 \Gamma_{2} & 1 & 0 & 0
\end{array}\right],} \\
& {\left[\begin{array}{ccc|c}
-2 \Gamma_{1} & -1 & 0 & 0 \\
1 & 0 & 0 & -\varepsilon
\end{array}\right],\left[\begin{array}{ccc|c}
2 \Gamma_{2} & 1 & 0 & 0 \\
1 & 0 & 0 & -\varepsilon
\end{array}\right] .}
\end{aligned}
$$

Define the residuals

$$
r=y-X \beta, \tilde{r}=\tilde{y}-\tilde{X} \tilde{\beta} .
$$

An equivalent formulation of the problem is

$$
\left[\begin{array}{ccc}
0 & 0 & A^{i} \\
0 & I & X \\
A^{i^{\top}} & X^{\top} & 0
\end{array}\right]\left[\begin{array}{l}
\lambda \\
r \\
\beta
\end{array}\right]=\left[\begin{array}{c}
b^{i} \\
y \\
0
\end{array}\right],
$$

where $\lambda$ is the Lagrange vector and $r$ is the residual. Defining

$$
\lambda=\tilde{\lambda}+\Delta \lambda, r=\tilde{r}+\Delta r, \beta=\tilde{\beta}+\Delta \beta .
$$

Notice that since $A^{i}=0$ and $b^{i}=0$, we have $r=0$ and $\lambda=0$, and the perturbed system can be written as

$$
\left[\begin{array}{ccc}
0 & 0 & \tilde{A}^{i} \\
0 & I & \tilde{X} \\
\tilde{A}^{\top} & \tilde{X}^{\top} & 0
\end{array}\right]\left[\begin{array}{c}
\tilde{\lambda} \\
\tilde{r} \\
\tilde{\beta}
\end{array}\right]=\left[\begin{array}{c}
\tilde{b}^{i} \\
\tilde{y} \\
0
\end{array}\right]
$$

Then we obtain

$$
\left[\begin{array}{ccc}
0 & 0 & \tilde{A}^{i} \\
0 & I & \tilde{X} \\
\tilde{A}^{\top} & \tilde{X}^{\top} & 0
\end{array}\right]\left[\begin{array}{c}
\Delta \lambda \\
\Delta r \\
\Delta \beta
\end{array}\right]=\left[\begin{array}{c}
\Delta b^{i}-\Delta A^{i} \beta \\
\Delta y-\Delta X \beta \\
-\Delta A^{i^{\top}} \lambda-\Delta X^{\top} r
\end{array}\right]
$$

With the help of the result in Cox and Higham [1999], we get

$$
\begin{aligned}
\Delta \beta= & \tilde{M}\left(\Delta b^{i}-\Delta A^{i} \beta\right)+(\tilde{X} \tilde{Q})^{\dagger}(\Delta y-\Delta X \beta) \\
& +\left((\tilde{X} \tilde{Q})^{\top} \tilde{X} \tilde{Q}\right)^{\dagger}\left(\Delta A^{i^{\top}} \lambda+\Delta X^{\top} r\right) .
\end{aligned}
$$


Recall that $\lambda=0$ and $r=0$. Thus,

$$
\Delta \beta=\tilde{M}\left(\Delta b^{i}-\Delta A^{i} \tilde{\beta}\right)+(\tilde{X} \tilde{Q})^{\dagger}(\Delta y-\Delta X \beta) .
$$

Also with the fact, $\Delta \beta$ is of order $\epsilon$. We therefore have

$$
\Delta \beta=\tilde{M}\left(\Delta b^{i}-\Delta A^{i} \tilde{\beta}\right)+(\tilde{X} \tilde{Q})^{\dagger}\left(\Delta y-\Delta X^{t} i l d e \beta\right)+O\left(\epsilon^{2}\right) .
$$

Taking 2-norms, we obtain

$$
\begin{aligned}
\|\Delta \beta\|_{2} \leq & \|\tilde{M}\|_{2}\left(\left\|\Delta b^{i}\right\|_{2}+\left\|\Delta A^{i}\right\|_{2}\|\tilde{\beta}\|_{2}\right) \\
& +\epsilon\left[\left\|(\tilde{X} \tilde{Q})^{\dagger}\right\|_{2}\left(\|\tilde{y}\|_{2}+\|\tilde{X}\|_{F}\|\tilde{\beta}\|_{2}\right)\right]+O\left(\epsilon^{2}\right) .
\end{aligned}
$$

Next step we consider the second case. We only need to set $\tilde{A}^{i}=0, \tilde{b}^{i}=0$, and $r=0$ in the expressions in the previous analysis. From (14), we know that

$$
\Delta \beta=\tilde{X}^{\dagger}(\Delta y-\Delta X \beta)
$$

Since $\Delta \beta$ is of order $\epsilon$, similarly, we get

$$
\Delta \beta=\tilde{X}^{\dagger}(\Delta y-\Delta X \tilde{\beta})+O\left(\epsilon^{2}\right)
$$

Taking 2-norms, we obtain

$$
\|\Delta \beta\|_{2} \leq \epsilon\left\|\tilde{X}^{\dagger}\right\|_{2}\left(\|\tilde{y}\|_{2}+\|\tilde{X}\|_{F}\|\tilde{\beta}\|_{2}\right)+O\left(\epsilon^{2}\right) .
$$

Based on the combination of two cases, we have

$$
\|\Delta \beta\|_{2} \leq \max \left\{\Sigma_{1}, \Sigma_{2}\right\}
$$

where $\Sigma_{1}$ and $\Sigma_{2}$ are given by Equations (16) and (19), respectively.

Theorem 3.1 gives the error bound of coefficients (i.e., $k, T_{0}$, and $P_{0}$ ). However, in a real system, we are more interested in the error bound of the clock skews predicted by Equation (1). Recall Equation (18); for each $i \in[1,3]$,

$$
\begin{aligned}
\Delta \beta_{i} & =\tilde{X}^{\dagger}(i,:) \Delta y-\tilde{X}^{\dagger} \Delta X \tilde{\beta}+O\left(\epsilon^{2}\right) \\
& \lesssim\left\|\tilde{X}^{\dagger}(i,:)\right\|_{2}\|\Delta y\|_{2}+\left\|\tilde{X}^{\dagger}\right\|_{2}\|\Delta X\|_{F}\|\tilde{\beta}\|_{2}:=a_{i} .
\end{aligned}
$$

Put them back to Equation (1); we have

$$
\Delta P_{T} \lesssim a_{1}(T-\bar{T})^{2}+a_{2}|T-\bar{T}|+a_{3},
$$

where $\bar{T}$ stands for the mean value of the temperatures in the training set $\Omega$.

Summary. As can be seen from Theorem 3.1, the bound of the estimation error $\Delta \beta$ is a function of $\psi_{1}$ and $\psi_{2}$, where $\psi_{1}$ and $\psi_{2}$ are the measurement errors of clock skews and temperatures, respectively. The smaller the measurement errors, the better the performance of TACO. Moreover, the error bound of predicted clock skews can also be deduced from Theorem 3.1, which is determined by the samples in the training set. We will validate this in Section 6 through our field test results.

\section{CLOCK SKEW MEASUREMENT}

In this section, we discuss how to measure clock skews of various temperatures.

\subsection{Overview of Skew Measurement}

Let $O^{s}(t)$ be the clock offset between node $s$ and the reference node $r$ at time $t$. Then the average clock skew $\bar{P}$ within $(t, t+\Delta t)$ is

$$
\bar{P}=\frac{O^{s}(t+\Delta t)-O^{s}(t)}{\Delta t} .
$$

If the temperature $T$ remains the same within that time window, we have $P_{T}=\bar{P}$. 
To obtain $t$ and $O^{s}(t)$, the reference node $r$ periodically broadcasts timestamped beacons, where the timestamp equals the reference time $t$. Once a sensor node $s$ receives a beacon, it records the arrival time $C^{s}(t)$ along with the timestamp $t$ and calculates the clock offset $O^{s}(t)$. With two consecutive beacons, node $s$ can estimate the clock skew. However, a few practical challenges are associated with this measurement.

Trade-off between the measurement accuracy and the temperature variation. The interval $\Delta t$ affects the measurement accuracy. Research [Schmid et al. 2009] has shown that the larger the interval, the higher the measurement accuracy. However, the large interval also increases the possibility of temperature variation, especially in an outdoor environment. If the temperature changes between two consecutive beacons, then $\bar{P}$ cannot be treated as $P_{T}$. To solve this problem, we propose a cumulative measurement method.

Message delay between the reference node and the sensor node. In reality, the arrival time at node $s$ is $C^{s}\left(t^{\prime}\right)$ but not $C^{s}(t)$, where $t^{\prime}-t$ is the message delay. The message delay contains various components and influences the measurement accuracy. To improve the accuracy, we measure the delay on the critical path to remove most uncertainties. A similar method is used by DMTS [Ping 2003].

In the following sections, we introduce cumulative measurement and critical path, respectively.

\subsection{Cumulative Measurement of Clock Skew}

To address the dilemma of the measurement accuracy and the temperature variation, we propose to calculate the average clock skew over cumulative measurements instead of at a specific temperature. This enables us to use a large $\Delta t$ to improve the measurement accuracy, regardless of whether temperature changes within $\Delta t$.

Given that the temperature is a function of time (i.e., $T(t))$, we have

$$
\begin{aligned}
& O^{s}(t+\Delta t)-O^{s}(t)=\int_{t}^{t+\Delta t} P^{s}(t) d t \\
& \quad=k \int_{t}^{t+\Delta t} T^{2}(t) d t-2 k T_{0} \int_{t}^{t+\Delta t} T(t) d t+\Delta t\left(P_{0}+k T_{0}^{2}\right) .
\end{aligned}
$$

Let $1 / \tau$ be the temperature sampling rate, and $\tau$ is chosen to be small enough (e.g., a few seconds) such that the temperature does not change between two consecutive sampling points. The preceding formula can be rewritten as

$$
\begin{aligned}
& O^{s}(t+\Delta t)-O^{s}(t) \\
& \quad=k \tau \sum_{i=1}^{\Delta t / \tau}(T[i])^{2}-2 k \tau T_{0} \sum_{i=1}^{\Delta t / \tau} T[i]+\Delta t\left(P_{0}+k T_{0}^{2}\right),
\end{aligned}
$$

where $T[i]$ is the $i$-th temperature sampled in the time slot $(t, t+\Delta t)$. Dividing both sides by $\Delta t$, we obtain the cumulative correlation:

$$
\bar{P}=k \bar{T}^{(2)}-2 k T_{0} \bar{T}^{(1)}+\left(P_{0}+k T_{0}^{2}\right),
$$

where

$$
\bar{T}^{(2)}=\frac{\tau \sum_{i=1}^{\Delta t / \tau}(T[i])^{2}}{\Delta t}
$$

and

$$
\bar{T}^{(1)}=\frac{\tau \sum_{i=1}^{\Delta t / \tau} T[i]}{\Delta t} .
$$




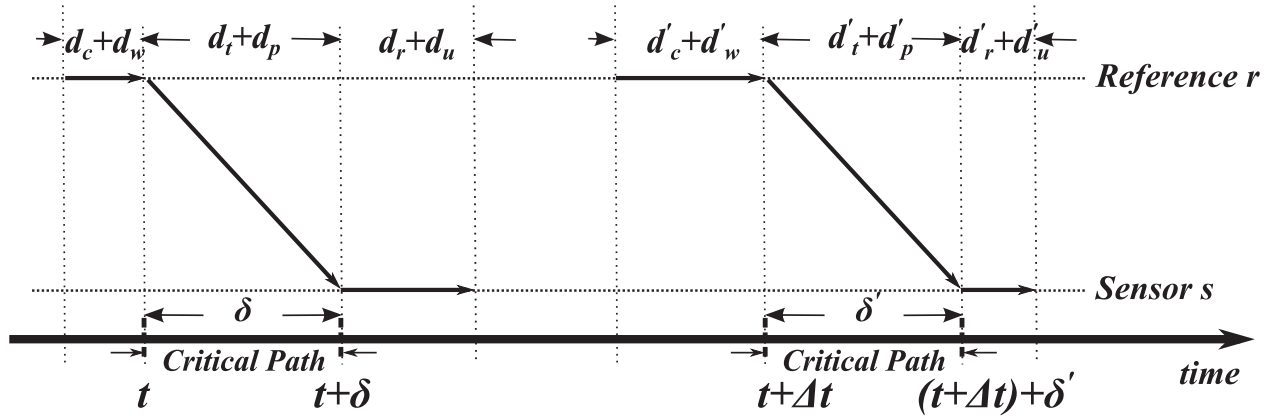

Fig. 5. Illustration of uncertainties lying in estimation of offset: (1) $d_{c}+d_{w}$ is the combination of send and access delay at reference $r ;(2) d_{t}+d_{p}$ is the combination of transmission and propagation delay over the air; and (3) $d_{r}+d_{u}$ is the combination of reception and processing delay at sensor $s$.

A node $s$ samples the temperature at a rate of $1 / \tau$ and calculates the average clock skew between consecutive beacons. Given $N$ continuous beacons, the node $s$ will obtain $N-1$ equations and have an updated linear model $y=X \beta$, where

$$
y=\left[\begin{array}{c}
\bar{P}_{1} \\
\vdots \\
\bar{P}_{N-1}
\end{array}\right] \text {, and } X=\left[\begin{array}{ccc}
\bar{T}_{1}^{(2)} & \bar{T}_{1}^{(1)} & 1 \\
\vdots & \vdots & \vdots \\
\bar{T}_{N-1}^{(2)} & \bar{T}_{N-1}^{(1)} & 1 .
\end{array}\right] \text {. }
$$

\subsection{Critical Path-Based Timestamp}

The accuracy of the average clock skew $\bar{P}$ is determined by the measurement of $O^{s}(t)$. In practice, we use the estimated offset $\hat{O}^{s}(t)$ instead, where $\hat{O}^{s}(t)=O^{s}(t)+\delta$ and $\delta$ is a packet delay. As existing research stated, the packet delay consists of the following delays [Ganeriwal et al. 2003]: sending delay $d_{c}$, channel access delay $d_{w}$, transmission delay $d_{t}$, propagation delay $d_{p}$, reception delay $d_{r}$, and receiving delay $d_{u}$.

To reduce the uncertainty of estimation errors, we utilize the critical path-based (CP-based) solution for timestamping, which is illustrated by Figure 5: the sender $r$ makes a timestamp right before a beacon is sent out, and the receiver $s$ makes another timestamp immediately after the start symbol arrives. As such, the estimation error $\delta$ only contains $d_{t}$ and $d_{p}$. Let $\hat{\bar{P}}$ be the estimated average clock skew between $t$ and $t+\Delta t$; then, the estimation error is

$$
\begin{aligned}
\hat{\bar{P}}-\bar{P} & =\frac{\hat{O}^{s}(t+\Delta t)-\hat{O}^{s}(t)}{\Delta t}-\frac{O^{s}(t+\Delta t)-O^{s}(t)}{\Delta t} \\
& =\frac{\delta^{\prime}-\delta}{\Delta t}=\frac{\left(d_{t}^{\prime}+d_{p}^{\prime}\right)-\left(d_{t}+d_{p}\right)}{\Delta t} .
\end{aligned}
$$

For fixed-length messages, $d_{t}$ is constant. Given that sensor nodes are stationary, $d_{p}$ is also constant. Therefore, the CP-based method improves the accuracy of $\bar{P}$. Such a method is similar to the one used in TPSN [Ganeriwal et al. 2003] and DMTS [Ping 2003].

\section{IMPLEMENTATION}

\subsection{Sensor Node Hardware}

We implement TACO on our customized marine sensor nodes (Figure 6). Each sensor node consists of a STM32F103RC low-power MCU (microcontroller), a TI TMP102 temperature sensor, a $512 \mathrm{kB}$ external flash for data storage, and an ATMEL AT86RF212 radio 


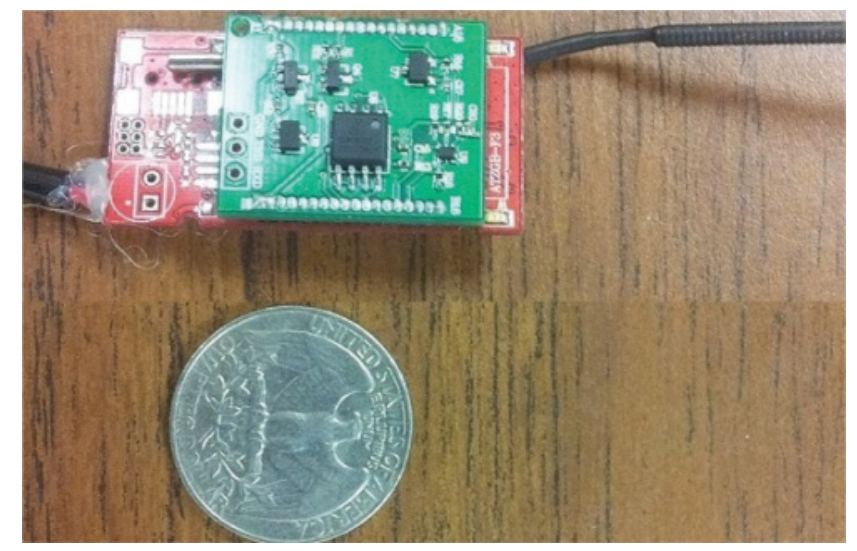

Fig. 6. Marine sensor node (battery excluded).

transceiver that supports IEEE 802.15.4. The system clock of the MCU is driven by a $16 \mathrm{MHz}$ external oscillator. Since the system clock is disabled when the MCU enters low-power mode, we use a $32.768 \mathrm{kHz}$ external oscillator, running all the time, to maintain the RTC. The RTC has a 32-bit register to count the ticks and provides a clock granularity of $30.5 \mu \mathrm{s}$.

\subsection{Implementation: Initialization Stage}

In the initialization stage, the sink node periodically broadcasts timestamped beacons. Each sensor node only accepts the incoming beacons from its parent and forwards the received beacons to its children. For any beacons that are from nodes other than its parent, the node will ignore them. For example, nodes at depth 1 will accept the beacons from the sink node and then forward the beacons to their children at depth 2 . Hop by hop, beacons from the sink node will spread over the entire tree. To avoid accumulating synchronization errors as the beacon travels from the sink to a node, the node will adjust the timestamp by adding the local elapsed ticks to the timestamp in the beacon. As a result, the synchronization errors from the parent to its children is at most one-hop errors.

Particularly in the initialization stage, each node will execute the algorithm summarized in Algorithm 1 to establish the correlation between the temperature and the clock skew. It measures the temperature every $\tau$ seconds and listens for timestamped beacons from its parent. Once a beacon from its parent arrives, the node checks whether the average temperature in the past window has been observed before. If this temperature has not yet been observed, the node calculates $\bar{P}, \bar{T}^{(1)}$, and $\bar{T}^{(2)}$ and forms an equation (as shown in Equation (22)). Here, we define that "a temperature has not been observed" if the new temperatures are not too close to any existing temperatures in the training set. This is enforced by the function TemperatureUnseen, which ensures that the difference between any two temperatures in the training set is larger than the sensors' measurement error (i.e., $0.5^{\circ} \mathrm{C}$ in our case). This procedure continues when the number of equations $N$ reaches a threshold $\gamma$, which ensures that the node will have enough training samples to establish the correlation. Theoretically, $\beta$ can be identified with three equations, but a larger number of equations is preferred to minimize the impact of measurement errors. However, a threshold that is too large will increase the overhead of LSQ-based computation and also the duration of the initialization stage. In our experiment, we choose a value between six to eight as the threshold because our field tests show that such values keep a good balance between the accuracy and the 


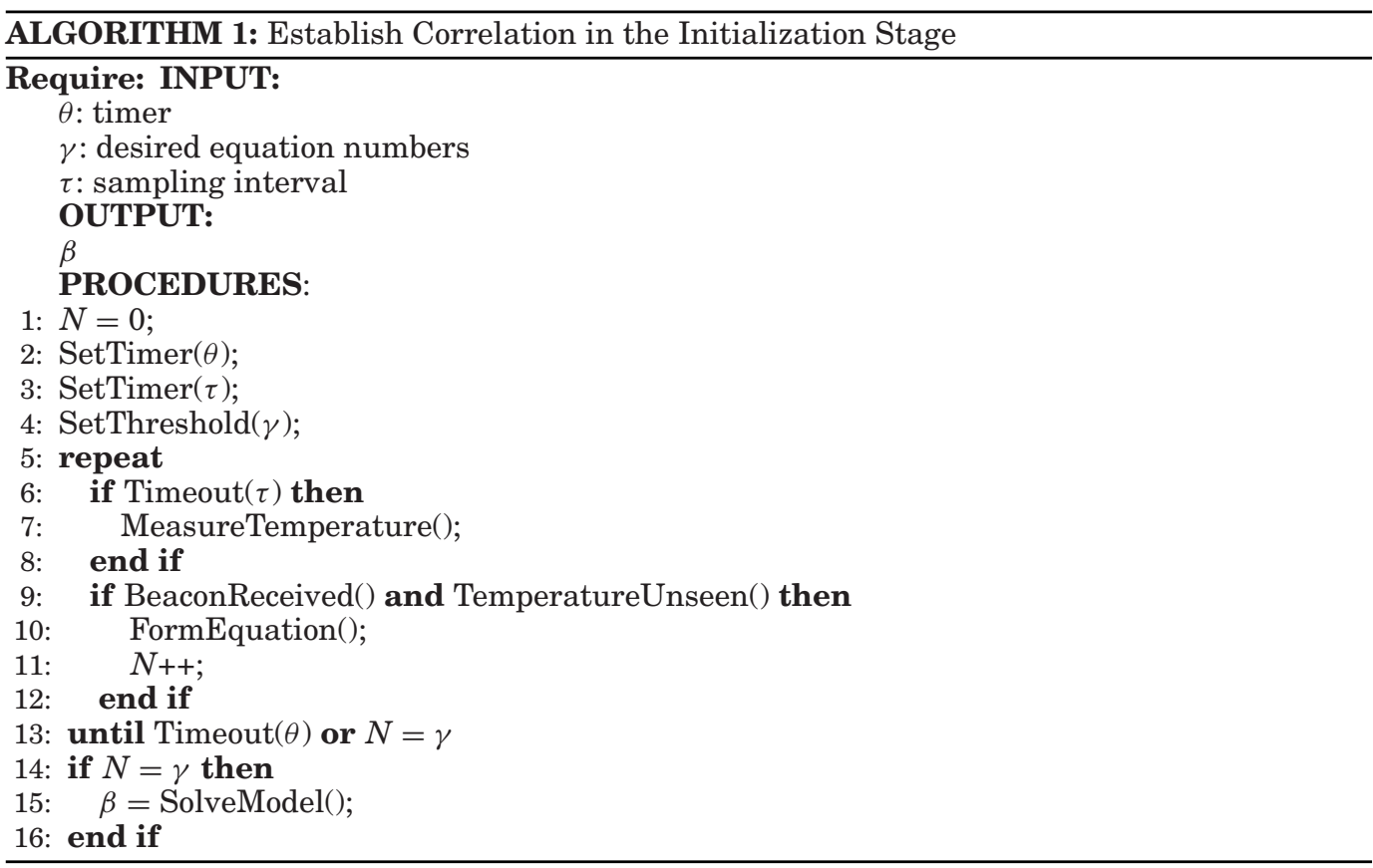

overhead. Once enough training samples are obtained, the node solves the constrained least squares problem and estimates the parameters (i.e., $k, T_{0}$, and $P_{0}$ ). Finally, the node will enter the compensation stage with the established correlation.

Mostly, a node will quit the initialization stage after obtaining enough training samples and establishing the correlation. However, in a deployment site where the environmental temperature rarely changes, it may take an unexpected long time to formulate enough equations. Therefore, a timer $\theta$ is set to enforce the termination of initialization in this situation. We will discuss this special case in Section 5.4.

\subsection{Implementation: Compensation Stage}

A node enters the compensation stage after the initialization. In the compensation stage, the node adjusts its local time periodically. Let $C^{s}\left(t_{i}\right)$ be the local time at the beginning of $i$-th time slot and $P^{s}\left(t_{i}\right)$ be the clock skew in the $i$-th time slot. Suppose that the duration of each time slot is $\zeta$. Then $C^{s}\left(t_{i+1}\right)$ can be calculated by

$$
\begin{aligned}
C^{s}\left(t_{i+1}\right) & =C^{s}\left(t_{i}\right)+\zeta\left(P^{s}\left(t_{i}\right)+1\right) \\
& =C^{s}\left(t_{1}\right)+\zeta \sum_{j=1}^{i}\left(P^{s}\left(t_{j}\right)+1\right) .
\end{aligned}
$$

Without loss of generality, we assume that node $s$ is synchronized with the reference at the beginning of the first time slot (i.e., $C^{s}\left(t_{1}\right)=t_{1}$ ). Thus, the adjusted local time $\hat{t}_{i}$ for the beginning of slot $i$ is

$$
\hat{t}_{i}=C^{s}\left(t_{i}\right)-\zeta \sum_{j=1}^{i-1} \hat{P}^{s}\left(t_{j}\right)
$$


where $\hat{P}^{s}\left(t_{j}\right)$ is the estimation of the clock skew in the $j$-th time slot. To obtain $\hat{P}^{s}\left(t_{j}\right)$, a node can sample the temperature $T$ at $t_{j}$ and calculate the clock skew $\hat{P}^{s}\left(t_{j}\right)$ based on the established correlation $P=P_{0}+k\left(T-T_{0}\right)$.

To summarize, in the compensation stage, each node adjusts its local time as follows: it measures the temperatures periodically, estimates the clock skews, and then adjusts the local time by following Equation (23).

\subsection{Special Cases}

Mostly, a node will obtain enough training samples in the initialization stage. However, in a deployment site where the environmental temperature rarely changes, it may take an unexpected long time for the node to formulate $\gamma$ equations. If this happens, it will break the node's regular functions. Thus, a timer must be set up in this special case to enforce the termination of the initialization stage. The timeout value $\theta$ can be decided by a network operator according to the deployment requirements. If a node fails to obtain $\gamma$ samples before the timer times out (i.e., $N<\gamma$ ), it will not establish the correlation in the initialization stage. Instead, the node considers the training set $\Omega=\left\{\left(P_{i}, T_{i}\right)\right\}$ as a look-up table. In this case, the table will be used temporarily to estimate the clock skew in the compensation stage [Schmid et al. 2009; Yang et al. 2012]. In the meantime, the node continues obtaining training samples. Once enough equations are formed, it identifies the correlation parameters by the LSQ-based method.

We envision that the LSQ-based computations need to be performed occasionally to overcome the impact of crystal aging. As mentioned in Section 3.1, a crystal oscillator's frequency will change over time due to aging, which usually occurs after 2 months and contributes up to $15 \%$ of frequency tolerance per year. As such, the long-term LSQbased computation must be performed occasionally at runtime to reflect this change. For example, it can be repeated once per month to update the correlation.

\subsection{Discussion}

Understanding clock skew $P_{0}$ and $P_{T}$. According to Equation (1), for a sensor node $s$, its clock skew $P_{T}$ consists of two components: the constant component $P_{0}$ and the dynamic component $k \times\left(T-T_{0}\right)^{2}$ that is caused by temperature variations. Considering a sensor network with a reference node whose clock is precise all the time, $P_{0}$ is the difference caused by nonidentical oscillator frequencies between the node $s$ and the reference node $r$ at the temperature $T_{0}$. Because this difference $P_{0}$ does not describe the dynamic component that varies with temperature changes, TACO provides better performance by employing $P_{T}$ instead of a constant calibration value $P_{0}$. We will validate this based on our field test result in Section 6.

Clock skews at the reference node. The reference node may be subject to clock skews caused by varying temperatures as well, leaving the timestamps in beacons different from the wall clock time. To address the issue, the reference node can use a TCXO. Alternatively, we can apply TACO to the reference node-the reference node estimates the correlation of its own clock skews and temperatures before the initialization stage using an accurate clock source such as GPS. During the initialization stage, the reference node will adjust its local time based on the environmental temperature and thus reduce the impact of its clock skews on other sensor nodes.

Initialization in the lab. The initialization stage for temperature-clock-skew correlation estimation can be conducted in a lab prior to deployment. However, the correlation of each node may change over time due to crystal aging, and the correlation estimation should be updated from time to time in the field during the compensation stage.

Topology dependency. TACO assumes that the routing protocol will form a tree automatically. Therefore, it does not start initialization until a tree rooted from a sink 
node is formed. Sensor nodes are installed during a low tide cycle when communication channels are available to ensure tree formation. However, TACO is independent of how a tree is formed. For example, it can employ the level discovery phase in TPSN [Ganeriwal et al. 2003] to create a hierarchical topology before the initialization stage or when a node's parent dies. In addition, TACO can work with existing tree-based synchronization protocols such as TPSN. The periodical SYNC messages in these protocols can serve as timestamped beacons in the compensation stage.

\section{EXPERIMENTS AND EVALUATION}

In this section, we evaluate the performance of TACO. We verify the CP-based clock skew estimation, examine the effectiveness of TACO, and compare TACO to a tablebased time synchronization algorithm.

\subsection{Accuracy of a CP-Based Timestamp}

The error analysis in Section 3 shows that the estimation error $\Delta \beta$ is bounded if measurement errors of temperature $T$ and clock skew $P$ are bounded. For the temperature $T$, it is usually measured by a temperature sensor. Its measurement accuracy is given and typically specified by the manufacturer. For example, for a TI TMP102 temperature sensor, its measurement error is within $0.5^{\circ} \mathrm{C}$. As a result, the accuracy of measuring clock skew $P$ is important to ensure the accuracy of $\beta$ (i.e., the temperature coefficients). To improve the accuracy of clock skew measurements $P$, we proposed the CP-based measurement method in Section 4. In this section, we validate the accuracy of the proposed method by comparing it toh a benchmark that measures clock skews directly using a data acquisition unit (DAQ) in the lab.

Figure 7(a) shows our experiment setup in the lab. We placed a marine sensor node in a temperature-controlled box (the box cover is not displayed in the figure) and a sink node under the room temperature. The sink node and the sensor node were powered by the same power supply. In the benchmark-based measurement, we wired each node's two pins to an NI USB-6009 DAQ [National Instruments 2012], which sampled the pin outputs at a frequency of $10 \mathrm{kHz}$. We set a timer for each node to toggle their pins once every 1 minute. Their pin outputs were captured and uploaded to a laptop. The uploaded data were processed by a Perl script, and the average clock skew was calculated.

In the meantime, we also let the sensor node run a CP-based measurement. The sink node broadcasted a timestamped beacon once every 5 minutes. Immediately after receiving the beacon, the sensor node calculated the clock offset $O^{s}(t)$ and uploaded it to the laptop, whereby the data were processed to calculate the average of CP-based clock skews.

We ran the experiments at two different temperatures independently: one at room temperature $\left(26^{\circ} \mathrm{C}\right)$ and the other at a higher temperature $\left(35^{\circ} \mathrm{C}\right)$. Each experiment lasted for 1 hour, and Table I summarizes the results. It shows that estimation errors between the CP-based measurement and the benchmark is less than $0.1 \%$.

\subsection{Performance of TACO}

To evaluate TACO, we deployed nine marine sensor nodes in an intertidal zone in Georgetown, South Carolina (Figure 1). Three of them were deployed on dry land, and the other six sensors were in the intertidal zone, resulting in a topology depicted in Figure 7(b). We also installed a sink node on dry land; this provided a reference time to the sensor nodes.

In the initialization stage, all sensor nodes sampled temperatures at an interval of $\tau=10 \mathrm{~s}$. The sink broadcasted the timestamped beacons once every $\omega=5$ minutes. In the compensation stage, all sensor nodes adjusted their local time at an interval 


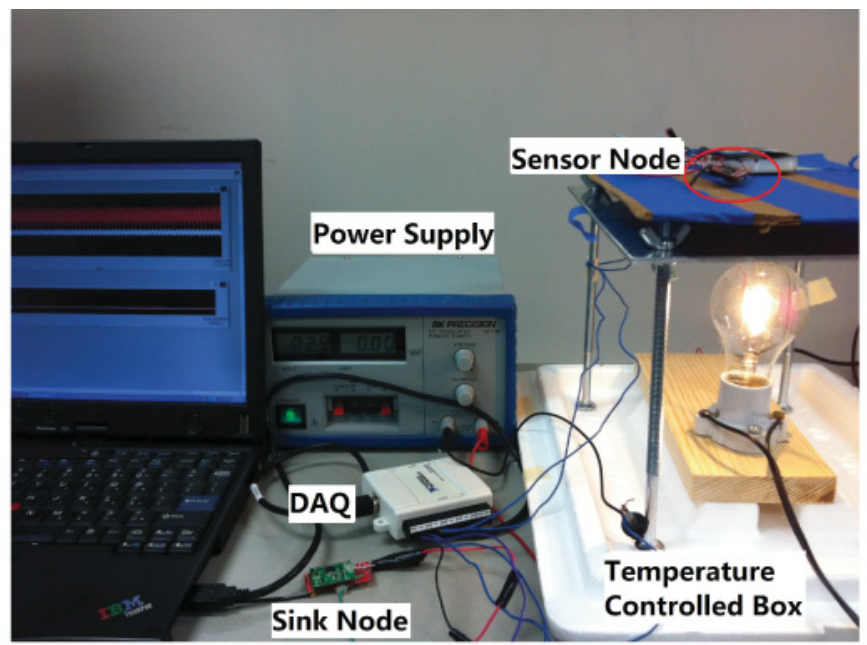

(a) Indoor setup

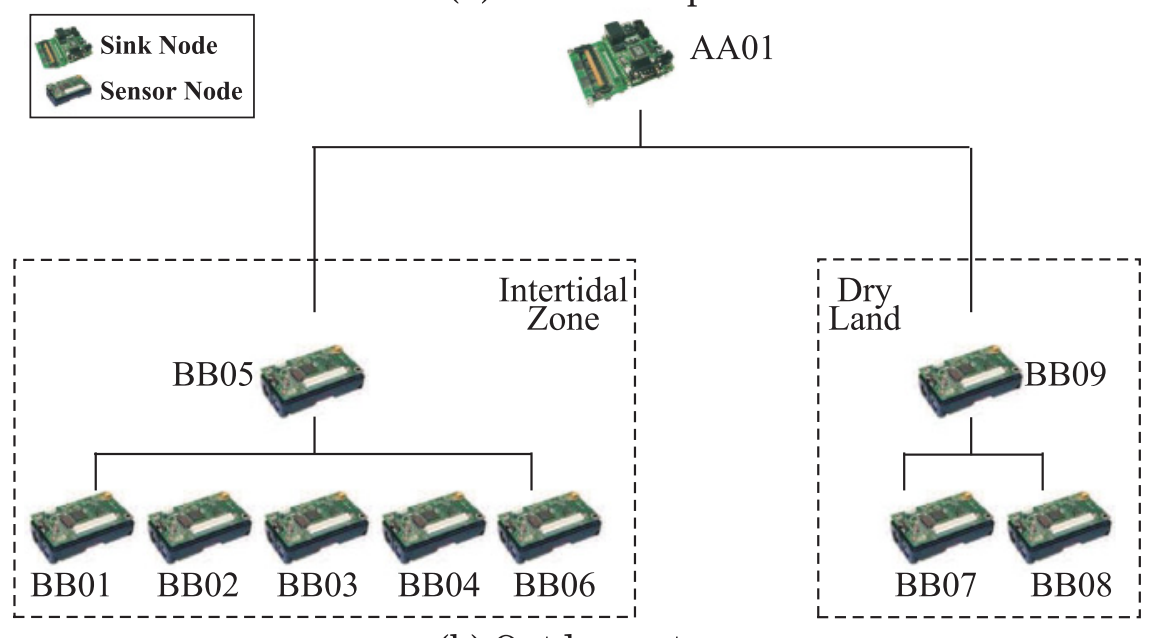

(b) Outdoor setup

Fig. 7. Experiment setup. (a) Indoor temperature controlled box (the box cover is not displayed here). (b) Outdoor deployment topology in an intertidal zone.

Table I. Clock Skew Measured by DAQ and the CP-Based Method

\begin{tabular}{|l|c|c|c|}
\hline Temperature & NI DAQ & CP Based & Error \\
\hline $26^{\circ} \mathrm{C}$ & $59.94 \mathrm{ppm}$ & $59.99 \mathrm{ppm}$ & $0.08 \%$ \\
\hline $35^{\circ} \mathrm{C}$ & $56.56 \mathrm{ppm}$ & $56.52 \mathrm{ppm}$ & $0.07 \%$ \\
\hline
\end{tabular}

of $\zeta=1$ minute. To evaluate the performance, we still let the sink node broadcast timestamped beacons once every 5 minutes in the compensation stage. These beacons were not used to synchronize nodes. Instead, they were used to record time drifts over time: upon receiving a timestamped beacon, all sensor nodes recorded their local time $C^{s}\left(t_{i}\right)$ that was not adjusted and the time $\hat{t}_{i}$ that was adjusted using TACO. Together with the timestamp contained in the beacon, these records were used to evaluate the following two metrics: 


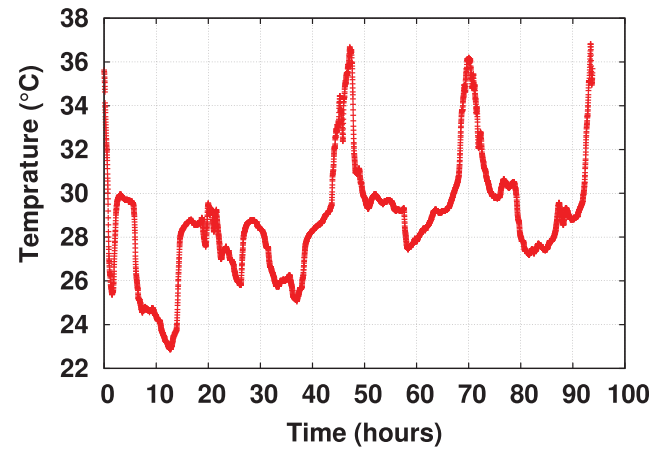

(a) Temperature

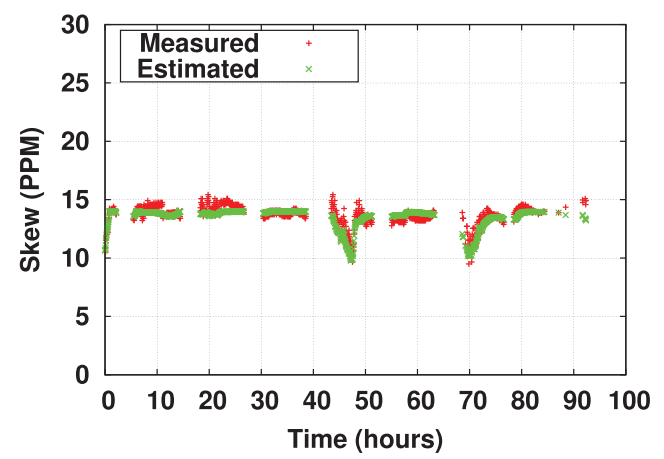

(c) Clock skews

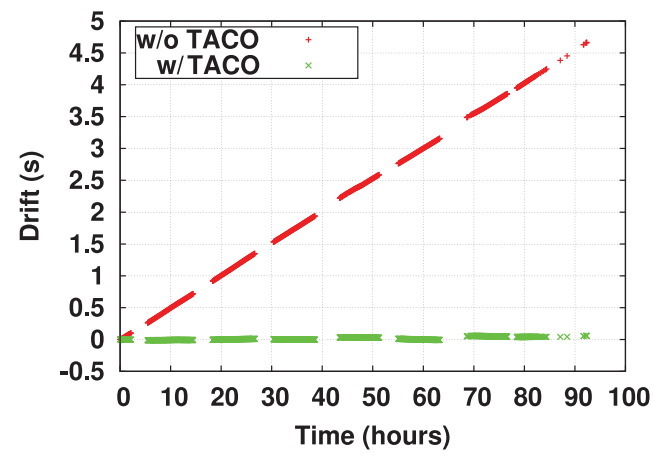

(b) Time drift

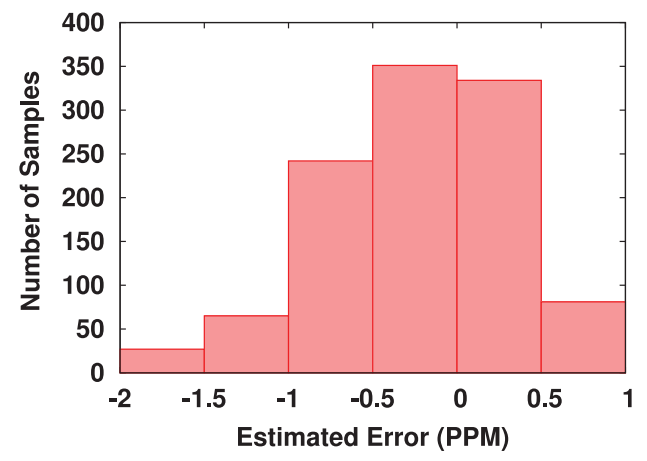

(d) Estimation errors

Fig. 8. Performance evaluation of TACO on node BB05. TACO can estimate the clock skews, with $92 \%$ of clock skew estimation errors less than $1 \mathrm{ppm}$. Given a temperature variation of $14^{\circ} \mathrm{C}$, the time drift is $4.6 \mathrm{~s}$. TACO effectively adjusts the local clock and reduces the time drift to $57 \mathrm{~ms}$.

-Time drift refers to the difference between the local time and the reference time. In our experiment, we collected the time drift of both the unadjusted local time and the adjusted local time. An ideal time adjustment algorithm should result in a time drift of zero.

- Skew estimation error is the error between the estimated skew $\hat{\bar{P}}_{s}(t)$ obtained by TACO (i.e., from the temperature-clock-skew correlation modeled by Equation (4.2)) and the measured clock skew $\bar{P}$ calculated based on reference times (contained in beacons) according to Equation (21). Both $\hat{\bar{P}}_{s}(t)$ and $\bar{P}$ are calculated between two beacons. We consider $\bar{P}$ as the comparison base because the CP-based timestamp exhibited high measurement accuracy in our first experiment.

To illustrate the performance of TACO, we present the data of a node (BB05) that is one hop to the sink node in Figure 8. Figure 8(a) shows that the temperature variation was about $14^{\circ} \mathrm{C}$. From the time drift depicted in Figure $8(\mathrm{~b})$, we observe that without local time adjustment, the time drift (in red) became larger than $4.6 \mathrm{~s}$ after 4 days, whereas the time drift (in green) was only $57 \mathrm{~ms}$ if TACO was used. Note that the lines in the plot are intermittent because no timestamped beacons were received due to the loss of communication during high tides; the clock drift continued to grow because sensor nodes did not adjust its local time according to beacons. In Section 3.4, Equation (20) shows that the error bound of predicted clock skews are determined by the training samples, especially by the mean value of temperatures in the training set. Based on this 


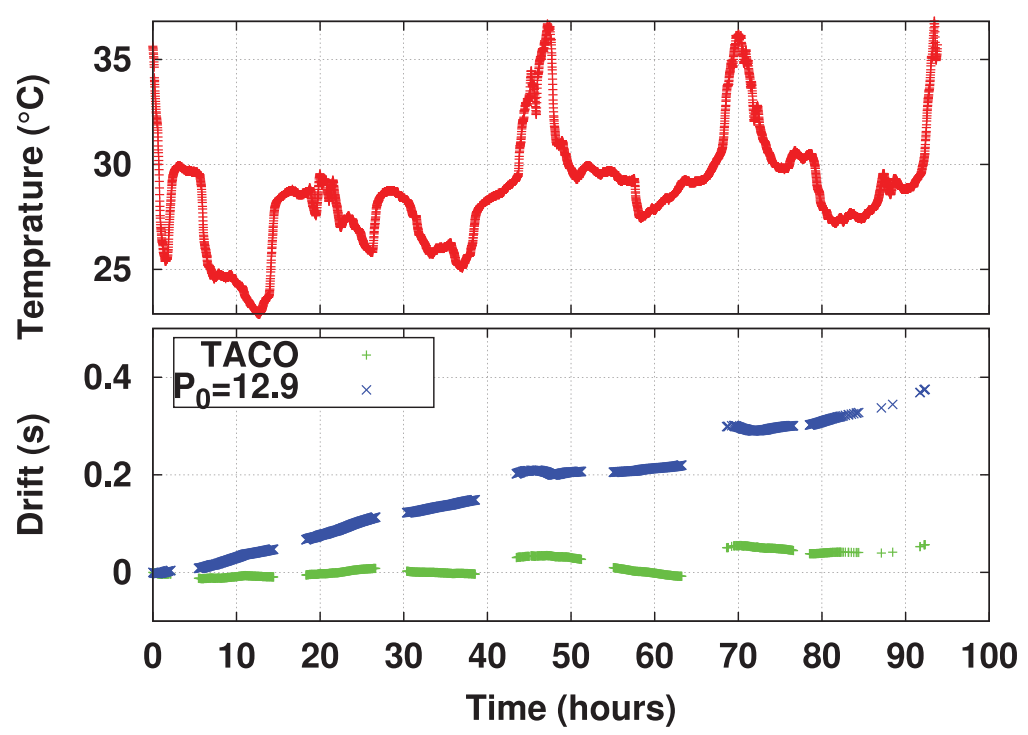

Fig. 9. Comparison between time compensated by TACO and by constant $P_{0}$. Using the constant value, the time drift was $375 \mathrm{~ms}$ after 4 days, whereas it was $57 \mathrm{~ms}$ using TACO.

equation and the training set of BB05, our calculation on the clock skew error bound has the following two findings: (1) BB05's upper bound between $25^{\circ} \mathrm{C}$ and $40^{\circ} \mathrm{C}$ will be between $0.12 \mathrm{ppm}$ and $2 \mathrm{ppm}$, and (2) its bound will become larger as the environmental temperature goes further from $33^{\circ} \mathrm{C}$. These two findings are validated by Figure 8(c) and (d). Figure 8(c) illustrates that the estimated clock skews (in green) approximate the measured clock skews (in red) closely. The maximum clock skew error is $2 \mathrm{ppm}$, and $92 \%$ of the estimation errors are less than $1 \mathrm{ppm}$ (Figure $8(\mathrm{~d})$ ), indicating that the correlation estimation is effective.

As discussed in Section 5.5, TACO provides better performance by employing $P_{T}$ rather than $P_{0}$. To verify this, we use our field test result and Matlab to simulate the scenario where only constant clock skew $P_{0}$ is considered. Figure 9 presents the result for node BB05, and it shows that TACO outperforms the compensation scheme that uses a constant value $P_{0}$ only. According to Figure 9 , if only $P_{0}$ is used, the time drift will be $375 \mathrm{~ms}$ after 4 days, which is 6.6 times the time drift by using $P_{T}$. Admittedly, in some cases, $P_{0}$ is a dominant component in $P_{T}$. However, by applying $P_{T}$, it still provides better results when temperature changes.

Figure 10(a) shows the overall performance of all deployed sensor nodes. Without TACO, the time drift ranged from 4.66s (node BB05) to 21.92s (node BB09). Most sensor node clocks drifted more than 10 s after 4 days. After applying TACO, all nodes' time drifts were reduced, with the highest drift being 591ms (node BB08). We also analyzed the skew estimation error for each sensor node, which is depicted in Figure 10(b). We observed that BB04, BB07, and BB08 have larger estimation errors than others, and thus their drifts after TACO are slightly larger (more than $470 \mathrm{~ms}$ ). This confirms our intuition: the larger the clock skew estimation errors, the less effective the time adjustment. In addition, the time drift between a sensor node and the sink node is influenced by the depth of a node: node BB05 and node BB09 were at depth 1, and they experienced smaller drift than their children. This can be explained by Theorem 3.1: the upper bound of the estimation error $\Delta \beta$ is a function of $\psi_{1}$ and $\psi_{2}$, where $\psi_{1}$ and $\psi_{2}$ are the measurement errors of clock skews and temperatures, respectively. The temperature error $\psi_{2}$ is independent of a node's depth, but the clock skew error 


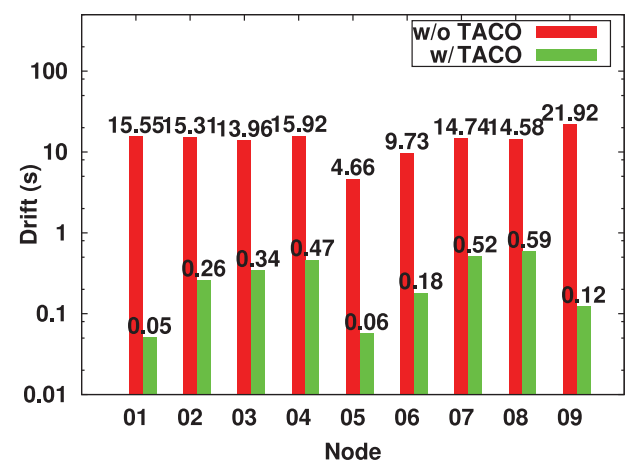

(a) Time drift

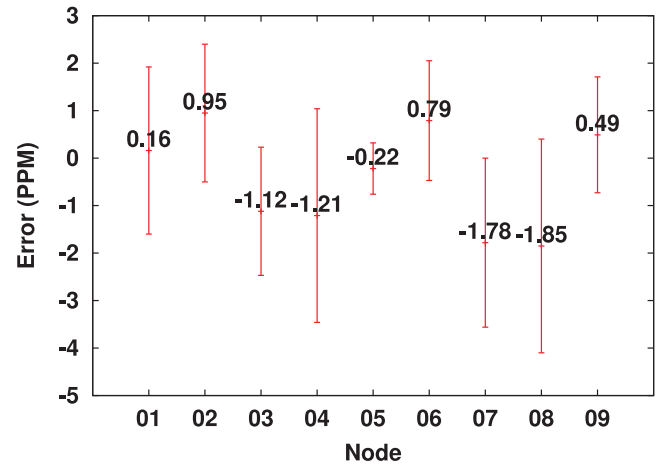

(b) Estimation errors

Fig. 10. Overall performance of TACO for all sensor nodes. (a) Time drift without and with TACO. (b) The mean and standard deviation of skew estimation errors during the compensation phase.

Table II. Absolute Estimation Errors Grouped by Different Hops

\begin{tabular}{|c|c|c|c|}
\hline & Tidal Zone & Dry Land & Average \\
\hline \hline 1 hop & $0.22 \mathrm{ppm}$ & $0.49 \mathrm{ppm}$ & $0.355 \mathrm{ppm}$ \\
\hline 2 hop & $0.85 \mathrm{ppm}$ & $1.82 \mathrm{ppm}$ & $1.335 \mathrm{ppm}$ \\
\hline
\end{tabular}

$\psi_{1}$ accumulates along the tree path. That is because as a node forwards beacons, it introduces additional uncertainty in time and thus induces the measurement errors of clock skews. We noticed that BB01 experienced less time drift compared to other children of BB05. It may be caused by the temperature differences, as its temperature readings in the training set were about $3^{\circ} \mathrm{C}$ higher on average than other nodes. Table II summarizes the absolute estimation errors grouped by hops, which confirms that the absolute error could accumulate along the path. Nevertheless, the key is to ensure communication between a node and its parent or children, and what really matters is the relative error.

\subsection{Comparison with Table-Based Schemes}

Prior work that exploits temperature [Schmid et al. 2009; Yang et al. 2012] for adjusting a local clock relies on a table of temperature and clock skew pairs. If a temperature is out of the range in the table, then message exchange is initiated to acquire the clock skew [Schmid et al. 2009]. If the reference node is unavailable, then a clock skew of the closest temperature in the table is selected instead [Yang et al. 2012].

To compare TACO to those table-based schemes, we simulated the table-based schemes using the temperature data and channel condition traces obtained in Georgetown, South Carolina. To construct the clock skew table, we identified all temperatures that were observable when the wireless channel was available (summarized in Figure 11(a)) and calculated the corresponding clock skews using the quadratic function (Equation (1)). Note that the temperature range of $27.30^{\circ} \mathrm{C}$ to $30.40^{\circ} \mathrm{Cwas}$ not observed during low tide periods and was not included in the table. Since the clock skews were calculated by Equation (1), the simulated table was not restricted by the granularity imposed by a real table-based scheme and could adjust the clock with a high granularity. Nevertheless, TACO outperforms the table-based scheme. Figure 11(b) summarizes the time drifts after different calibration schemes are applied, including TACO, table based, and constant value based. The result shows that the time drift of the tablebased scheme is about $168 \mathrm{~ms}$ after 4 days-two times larger than the drift of TACO 


\begin{tabular}{|l|l|}
\hline Overall & $22.88^{\circ} \mathrm{C} \sim 36.81^{\circ} \mathrm{C}$ \\
\hline $\begin{array}{l}\text { In the air } \\
\text { (observed) }\end{array}$ & $\begin{array}{l}22.88^{\circ} \mathrm{C} \sim 27.30^{\circ} \mathrm{C} \\
30.40^{\circ} \mathrm{C} \sim 36.81^{\circ} \mathrm{C}\end{array}$ \\
\hline Submerged & $25.85^{\circ} \mathrm{C} \sim 31.20^{\circ} \mathrm{C}$ \\
\hline $\begin{array}{l}\text { Non- } \\
\text { observed }\end{array}$ & $27.30^{\circ} \mathrm{C} \sim 30.40^{\circ} \mathrm{C}$ \\
\hline
\end{tabular}

(a) Temperature range of node BB05

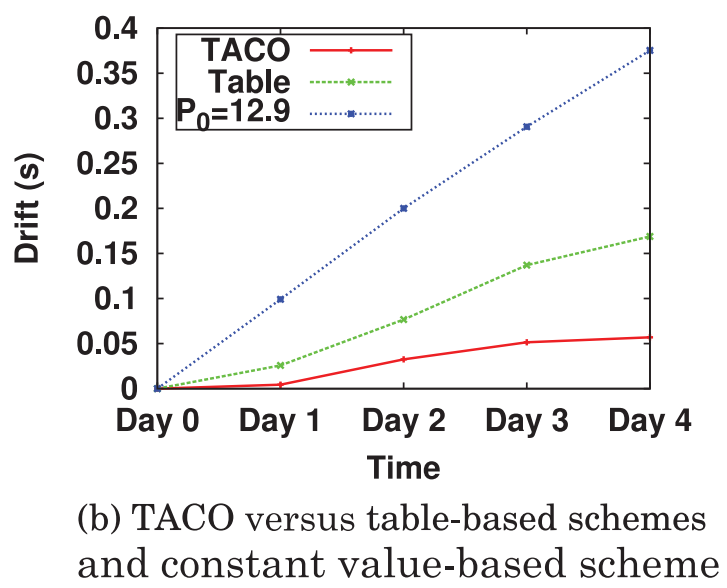

Fig. 11. Comparison among different calibration schemes. (a) Temperature range. Any skew at a temperature in the nonoverlap range is set to the skew at a close temperature. (b) Time drift after applying TACO, table-based scheme, and constant value-based $\left(\mathrm{P}_{0}=12.9\right)$ scheme.

( $57 \mathrm{~ms})$. This is because table-based schemes cannot predict an accurate clock skew for a temperature that had not been observed (i.e., $27.30^{\circ} \mathrm{C}$ to $30.40^{\circ} \mathrm{C}$ ).

\section{ENERGY MODELING AND ANALYZING}

We propose TACO because its local compensation scheme increases the required time interval of resynchronization and thus saves energy consumed by broadcasting SYNC messages. This energy saving, however, is achieved at the cost of periodical temperature measurement.

In this section, we ask this question: can we really save energy by performing TACO? In particular, we consider the following two network scenarios: $S 1$, a network whose time synchronization purely relies on broadcasting SYNC messages, and $S 2$, a network whose time synchronization relies on a combination of broadcasting SYNC messages and performing TACO. Given a time window $\Gamma$, let $E_{\Gamma_{1}}$ and $E_{\Gamma_{2}}$ be the energy consumption in scenarios $S 1$ and $S 2$, respectively; the question is equivalent to to achieve the same level of synchronization accuracy, in which condition will $E_{\Gamma_{2}}<E_{\Gamma_{1}}$ hold?

To tackle this problem, we start the analysis by formulating a general energy model that can be applied to TACO and other temperature-aware compensation schemes. We then apply the energy model into the preceding network scenarios and show that $S 2$ consumes less energy than $S 1$ in most cases.

\subsection{Energy Model}

In this section, we formulate a general energy model for time synchronizations. We assume that each component of the node can be turned on or turned off separately. For 
example, the node can turn off its radio transceiver when it measures the environmental temperature using the temperature sensor. Similarly, the temperature sensor can be turned off when the node transmits SYNC messages. As such, given a time window $\Gamma$, the energy used by time synchronizations (denoted by $E_{\Gamma}$ ) consists of the following parts: (1) the energy $E_{\mathrm{M}}$ used by communications for SYNC messages, (2) the energy $E_{\mathrm{T}}$ for temperature sensing, and (3) the energy $E_{\mathrm{L}}$ for computation overhead. In other words,

$$
E_{\Gamma}=E_{\mathrm{M}}+E_{\mathrm{T}}+E_{\mathrm{L}} .
$$

We first consider $E_{\mathrm{M}}$. A node periodically turns on its radio chip to receive SYNC messages and forward them to its children. Let $n_{\mathrm{M}}$ be the number of times that a radio is turned on in the time window $\Gamma$, and let $e_{\mathrm{M}}$ be the average energy that is used each time the radio is on; thus,

$$
E_{\mathrm{M}}=e_{\mathrm{M}} \times n_{\mathrm{M}} .
$$

Assume that $\omega$ is the time interval of periodical SYNC messages; then,

$$
n_{\mathrm{M}}=\frac{\Gamma}{\omega} \text {. }
$$

For $e_{\mathrm{M}}$, it includes the energy used by idle listening, receiving, and transmitting messages [Polastre et al. 2004; Wu et al. 2012]. In addition, considering overheard messages and retransmitted messages, we can formulate $e_{\mathrm{M}}$ as

$$
e_{\mathrm{M}}=e_{\text {idle }}+\left(q_{1}+1\right) \times e_{\mathrm{rx}}+\left(q_{2}+1\right) \times e_{\mathrm{tx}} .
$$

Here, $e_{\mathrm{rx}}$ and $e_{\mathrm{tx}}$ are the energy to receive and transmit one SYNC message, and $q_{1}$ and $q_{2}$ are coefficients that reflect the impact of overheard messages and retransmitted messages.

For the energy $E_{\mathrm{T}}$, we have

$$
E_{\mathrm{T}}=e_{\mathrm{T}} \times n_{\mathrm{T}},
$$

where $e_{\mathrm{T}}$ is the energy used by the temperature sensor for a single measurement and $n_{\mathrm{T}}$ is the number of measurements in time window $\Gamma$.

$E_{\mathrm{L}}$ is the energy used by computation overhead, which depends on the implementation. We assume that the computation can be divided into different tasks (e.g., LSQ computation), and each task is repeated periodically. Therefore, we have

$$
E_{\mathrm{L}}=\sum_{i} e_{L_{i}} \times n_{L_{i}}
$$

where $e_{L_{i}}$ is the energy of a single operation of task $i$ and $n_{L_{i}}$ is the repeated number of task $i$ in time window $\Gamma$.

Putting them together, we finally have the following energy model:

$$
\begin{aligned}
E_{\Gamma} & =E_{\mathrm{M}}+E_{\mathrm{T}}+E_{\mathrm{L}} \\
& =e_{\mathrm{M}} \times n_{\mathrm{M}}+e_{\mathrm{T}} \times n_{\mathrm{T}}+\sum_{i} e_{L_{i}} \times n_{L_{i}} .
\end{aligned}
$$

\subsection{Energy Analysis}

Consider the two network scenarios proposed at the beginning of this section: $S 1$, a network that purely relies on SYNC messages (i.e., $n_{\mathrm{T}}=0$ and $n_{\mathrm{L}}=0$ ), and $S 2$, a network that relies on the combination of SYNC messages and TACO. Let $E_{\Gamma_{1}}$ and $E_{\Gamma_{2}}$ be the energy in scenarios $S 1$ and $S 2$, respectively. Intuitively, performing local compensation 
Table III. Typical Values of Marine Nodes

\begin{tabular}{|l|c|c|c|}
\hline Component & Current Drawn & Duration & Energy $(\mathrm{mA} \cdot \mathrm{s})$ \\
\hline \hline TMP & $0.01 \mathrm{~mA}$ & $26 \mathrm{~ms}$ & $e_{\mathrm{T}}=2.6 \times 10^{-4}$ \\
\hline COMP & $12.8 \mathrm{~mA}$ & - & - \\
\hline RADIO & $9.2 \mathrm{~mA}(\mathrm{Idle})$ & $20 \mathrm{~ms}$ & \multirow{2}{*}{$e_{\mathrm{M}}=0.37$} \\
\hline & $25 \mathrm{~mA}(\mathrm{TX} / \mathrm{RX})$ & & \\
\hline
\end{tabular}

Note: The duration of COMP (computation) depends on the implementation.

increases the time interval of transmitting SYNC messages, which reduces $n_{\mathrm{M}}$. Therefore, the energy $E_{\mathrm{M}}$ used by SYNC messages in scenario $S 2$ is reduced. This goal is achieved at the cost of increasing $E_{\mathrm{T}}$. However, given that a sensing component usually consumes less energy than a communication component, we should have $E_{\Gamma_{2}}<E_{\Gamma_{1}}$.

Table III summarizes a marine node's typical values. The energy $e_{\mathrm{M}}$ is more complicated because it is impacted by two factors: (1) $q_{1}$, the number of overheard messages due to nonparent neighbors, and (2) $q_{2}$, the number of retransmitted messages due to collisions. To simplify the discussion, we consider the best case where no retransmission happens (i.e., $q_{2}=0$ ) and no messages are overheard (i.e., $q_{1}=0$ ). A SYNC message is 30 bytes long (including preamble and frame checksum), and the data rate is 40kbps; therefore, receiving a SYNC message lasts $6 \mathrm{~ms}$. Likewise, forwarding the SYNC message also lasts $6 \mathrm{~ms}$. Moreover, to successfully transmit and receive a SYNC message between a parent and a child, the radio chip must be turned on at least twice the desired accuracy bound. For example, if the desired worst-case accuracy bound between any pair of neighbors is $10 \mathrm{~ms}$, then a node must turn on its radio at least $20 \mathrm{~ms}$. As a result,

$$
e_{\mathrm{M}}=9.2 \times(0.020-2 \times 0.006)+25 \times 0.006+25 \times 0.006 \doteq 0.37 \mathrm{~mA} \cdot \mathrm{s} .
$$

Take node BB05, for instance. To achieve a 10ms accuracy bound, a SYNC message must be broadcast once per 12.6 minutes in $S 1$, whereas it must be broadcast once per 16.8 hours in $S 2$. Let the time window $\Gamma$ be 1 month; then, for scenario $S 1, n_{\mathrm{M}}=3,428$ SYNC messages will be generated and the total energy consumption is

$$
E_{\Gamma_{1}}=0.37 \times 3428+0+0=1268.36 \mathrm{~mA} \cdot \mathrm{s} .
$$

In $S 2$, only $n_{\mathrm{M}}=43 \mathrm{SYNC}$ messages are generated within 1 month, so

$$
E_{\mathrm{M}}=0.37 \times 43=15.91 \mathrm{~mA} \cdot \mathrm{s} .
$$

We assume that the temperature sensing is performed once per minute, which leads to

$$
E_{\mathrm{T}}=2.6 \times 10^{-4} \times 43200=11.23 \mathrm{~mA} \cdot \mathrm{s} .
$$

The computation overhead includes two major tasks. First, after each temperature sensing, the node predicts clock skew based on Equation (1), which introduces $2.56 \times$ $10^{-5} \mathrm{~mA} \cdot \mathrm{s}$ energy cost each time. Second, to reflect crystal aging at runtime, the node performs LSQ calculations once per month to update the correlation, which lasts $0.8 \mathrm{~ms}$ for each calculation. As a result,

$$
E_{\mathrm{L}}=2.56 \times 10^{-5} \times 43200+12.8 \times 0.0008 \times 1=1.12 \mathrm{~mA} \cdot \mathrm{s} .
$$

Thus, the total energy cost in $S 2$ is

$$
E_{\Gamma_{2}}=E_{\mathrm{M}}+E_{\mathrm{T}}+E_{\mathrm{L}}=28.26 \mathrm{~mA} \cdot \mathrm{s},
$$

which is only $2.2 \%$ of $E_{\Gamma_{1}}$. 
Table IV. Energy Consumption of Marine Nodes Used by Different Time Synchronization Schemes

\begin{tabular}{|l|c|c|}
\hline Pure Messages & SYNC + TACO & SYNC + Table Based \\
\hline $1268.36 \mathrm{~mA} \cdot \mathrm{s}$ & $28.26 \mathrm{~mA} \cdot \mathrm{s}$ & $57.85 \mathrm{~mA} \cdot \mathrm{s}$ \\
\hline
\end{tabular}

\subsection{Model Discussion}

The energy model proposed here can be applied to other temperature-compensated protocols such as table-based protocols, because the model is independent of how compensation is performed. Similar to TACO, in a table-based protocol, total energy consumption $E_{\Gamma}$ is reduced by performing local temperature-based calibrations instead of frequent message exchange $E_{\mathrm{M}}$. For instance, consider the table-based protocol discussed in Section 6.3. According to our simulation result, a SYNC message must be broadcast once every 5.7 hours to achieve an accuracy of $10 \mathrm{~ms}$. In other words, only 126 SYNC messages are generated within 1 month. Assume that the temperature sampling rate is the same with TACO and the table look-up algorithm is implemented efficiently such that $E_{\mathrm{L}}$ can be ignored. Let $E_{\Gamma_{3}}$ be the total energy of the table-based protocol; then,

$$
E_{\Gamma_{3}}=0.37 \times 126+2.6 \times 10^{-4} \times 43200=57.85 \mathrm{~mA} \cdot \mathrm{s},
$$

which is only $4.6 \%$ of $E_{\Gamma_{1}}$ but slightly larger than $E_{\Gamma_{2}}$. Table IV summarizes the energy used by time synchronizations in different scenarios.

To summarize, the energy model describes the total consumption of time synchronizations. It consists of the major energy costs including communications, sensing, and computations. Moreover, utilizing this model, we show that TACO saves the energy by reducing a large portion of SYNC messages. We note that this model does not consider minor overhead such as radio ramp-up and carrier sensing, which will be our future work.

\section{RELATED WORK}

The most widely adopted time synchronization protocol on the Internet is NTP [Mills 1991], whereby a set of NTP packets that contain timestamps of the departure and arrival time are exchanged between the primary NTP server and client for synchronization. NTP provides an accuracy within a few milliseconds, but it is not suitable for WSNs because of its high synchronization overhead.

Many broadcast-based synchronization protocols for WSNs have been proposed [Elson et al. 2002; Elson and Estrin 2003; Ganeriwal et al. 2003; Maróti et al. 2004; Ping 2003; Yildirim and Kantarci 2014; Ferrari et al. 2011]. Elson et al. [2002] proposed RBS, in which neighboring nodes synchronize with each other by exploiting the fact that they receive the same synchronization message at approximately the same time. Removing the uncertainty at the sending path from the critical path, RBS achieves better accuracy than NTP. In their later work [Elson and Estrin 2003], the authors proposed a low-power synchronization scheme-postfactor synchronization. In this scheme, a "third-party" node only broadcasts a synchronization pulse to her neighbors when a stimulus arrives, and nodes that receive the pulse use it as a reference to their local stimulus timestamps. Postfactor synchronization is energy efficient for those beamforming applications, but it is inappropriate for long-distance communication scenarios. TPSN [Ganeriwal et al. 2003] consists of a level discovery phase and a synchronization phase. Similar to NTP, TPSN uses four timestamps to calculate the time offset and the delay across the entire networks. FTSP [Maróti et al. 2004] works similarly to TPSN, but it improves on TPSN and can cope well with link and node failure. DMTS [Ping 2003] is based on the estimation of all delays on the message transmission path. It exploits the concepts of event timestamp and network event scheduling to remove 
uncertainties lying in the message delay. Glossy decouples flooding from other network activities and exploits constructive interference for fast flooding [Ferrari et al. 2011]. These message broadcast-based schemes provide network-wide time synchronization and work well in large-scale sensor networks. However, they rely on synchronization broadcast and are inapplicable to WSNs with intermittent wireless channels.

ODS [Zhong et al. 2011] introduces on-demand synchronization to reduce the communication overhead. It adaptively adjusts the resynchronization interval given the required clock accuracy. ODS still relies on wireless channels for synchronization and does not provide any local time compensation mechanism.

Liu et al. focus on time synchronization for mobile underwater sensor networks [Liu et al. 2013, 2014]. Mobi-Sync utilizes the spatial correlation of mobile nodes for the propagation delay estimation [Liu et al. 2013]. DA-Sync estimates the Doppler shift caused by mobility [Liu et al. 2014]. As a result, these schemes do not suffer but benefit from nodes' mobility. Compared to Mobi-Sync and DA-Sync, TACO is designed with a special interest in WSNs in inter-tidal zones, where nodes can alternately be underwater and above water.

Several works focus on modeling clock skews and adjust clock drift to extend the resynchronization periods. Wu et al. [2011] consider clock synchronization under a statistical signal processing framework. They point out that clock synchronization problem is inherently related to parameter estimation. As a result, an efficient synchronization scheme should rely on accurate estimation of clock offset and skew. RBS [Elson et al. 2002] and FTSP [Maróti et al. 2004] model clock skew as a constant and use linear regression for adjusting clock drift. Several approaches [Hamilton et al. 2008; Bletsas 2003; Auler and d'Amore 2007] utilize the Kalman filter to track the clock offset and skew, and they assume that clock skew is a random process with zero mean. Leng and $\mathrm{Wu}$ [2010] utilize a maximum-likelihood estimator to estimate clock skew with unknown synchronization delay. In their later work [Leng and Wu 2011], they propose a fully distributed clock synchronization algorithm based on belief propagation [Leng and $\mathrm{Wu} 2011]$. These approaches treat clock skew as a random process. In comparison, our work identifies the dominant underlying factor (i.e., temperature) affecting clock skew and utilizes the correlation to adjust clock drift.

Consensus-based synchronization protocols are robust to node failures and network dynamics. ATS [Schenato and Fiorentin 2011] synchronizes clock speeds and clock offsets separately in two steps using the cascade of two consensus algorithms. Maggs et al. also propose a consensus clock synchronization scheme that provides internal synchronization to a virtual clock for WSNs [Maggs et al. 2012]. However, to compensate for the skew differences among nodes, existing consensus-based clock synchronization protocols require sufficient transmissions between nodes. Compared to consensus-based protocols, TACO does not rely much on communication between nodes once the parameters in the LSQ model are determined. As a result, TACO can save the energy consumed by wireless communications.

To the best of our knowledge, EACS [Schmid et al. 2009] and TCTS [Yang et al. 2012] are the first works proposed to compensate clock drift based on environmental temperature in a WSN. Both schemes build a calibration table that maps different temperatures to their associated clock skews at the initialization stage. During the running time, a sensor node adjusts its clock by looking up the compensation value in the table. If the temperature is out of range in the table, the node either triggers a message exchange to obtain a new clock skew [Schmid et al. 2009] or uses a bound value [Yang et al. 2012]. Such methods are not always applicable to WSN-IT, as channels may not be available when the temperature is out of range. TACO differs from their work in the following aspects. First, it relies on a quadratic function rather than an established calibration table. Therefore, TACO can predict a clock skew at a temperature that falls out of the 
table created in the initialization stage. Second, consider a sensor network that will experience summer and winter. TACO can establish the correlation in less than 1 hour, but EACS and TCTS cannot establish a table that contains all temperatures in such a short time unless the initialization is conducted in a temperature-controlled condition, which may not be available for all users. Last but not least, even for EACS and TCTS, TACO is useful: it can help them build a calibration table quickly by creating a sufficient number of elements by utilizing the established correlation. Such advantages are important in an outdoor WSN whose wireless channels are intermittent.

\section{CONCLUSION}

This work studied the time synchronization problem in WSNs deployed in intertidal zones (WSN-IT). The existing synchronization algorithms that rely on frequent resynchronization message exchange are inapplicable to WSN-IT, because the periodic tidal cycles could cause wireless channels to be unavailable for up to 24 hours. We proposed to model the clock skews as a quadratic function of temperature and designed TACO. TACO estimates the correlation between temperatures and clock skews by solving a constrained least square problem and continuously adjusts the local time based on environmental temperatures. We conducted experiments in an intertidal zone and have shown that TACO improves clock accuracy. As a direction for future work, we will investigate the influence of aged crystals on our synchronization scheme.

\section{REFERENCES}

Leandro Fabricio Auler and Roberto d'Amore. 2007. Adaptive Kalman filter for time synchronization over packet-switched networks: An heuristic approach. In Proceedings of the IEEE COMSWARE Conference. $1-7$.

Aggelos Bletsas. 2003. Evaluation of Kalman filtering for network time keeping. In Proceedings of the PERCOM Conference. 1452-1460.

Lloyd Butler. 1987. Underwater radio communication. In Amateur Radio.

A. J. Cox and N. J. Higham. 1999. Accuracy and stability of the null space method for solving the equality constrained least squares problem. BIT Numerical Mathematics 39, 1, 34-50.

Jeremy Elson, Lewis Girod, and Deborah Estrin. 2002. Fine-grained network time synchronization using reference broadcasts. SIGOPS Operating Systems Review 36, SI, 147-163.

Jeremy Eric Elson and Deborah Estrin. 2003. Time Synchronization in Wireless Sensor Networks. Ph.D. Dissertation. University of California, Los Angeles.

Epson. 2014. Epson MC-146/MC-156 Crystal Unit Data Sheet. Available at http://www.epsondevice.com.

Federico Ferrari, Marco Zimmerling, Lothar Thiele, and Olga Saukh. 2011. Efficient network flooding and time synchronization with glossy. In Proceedings of the IPSN Conference. 73-84.

R. Fletcher. 1971. A general quadratic programming algorithm. IMA Journal of Applied Mathematics 7, 1, 76-91.

Saurabh Ganeriwal, Ram Kumar, and Mani B. Srivastava. 2003. Timing-sync protocol for sensor networks. In Proceedings of the SenSys Conference. 138-149. DOI : http://dx.doi.org/10.1145/958491.958508

Donald Goldfarb. 1972. Extensions of Newton's method and simplex methods for solving quadratic programs. In Numerical Methods for Nonlinear Optimization, F. Lootsma (Ed.). Academic Press, London, England, 239-254.

D. Goldfarb and A. Idnani. 1983. A numerically stable dual method for solving strictly convex quadratic programs. Mathematical Programming, 1, 1-33.

Benjamin R. Hamilton, Xiaoli Ma, Qi Zhao, and Jun Xu. 2008. ACES: Adaptive clock estimation and synchronization using Kalman filtering. In Proceedings of the MobiCom Conference. 152-162.

Carl Hartung, Richard Han, Carl Seielstad, and Saxon Holbrook. 2006. FireWxNet: A multi-tiered portable wireless system for monitoring weather conditions in wildland fire environments. In Proceedings of the MobiSys Conference. 28-41. DOI : http://dx.doi.org/10.1145/1134680.1134685

C. S. Lam. 2008. A review of the recent development of MEMS and crystal oscillators and their impacts on the frequency control products industry. In Proceedings of the IEEE Ultrasonics Symposium. 694-704.

Mei Leng and Yik-Chung Wu. 2010. On clock synchronization algorithms for wireless sensor networks under unknown delay. IEEE Transactions on Vehicular Technology 59, 1, 182-190. 
Mei Leng and Yik-Chung Wu. 2011. Distributed clock synchronization for wireless sensor networks using belief propagation. IEEE Transactions on Signal Processing 59, 11, 5404-5414.

Jun Liu, Zhaohui Wang, Michael Zuba, Zheng Peng, Jun-Hong Cui, and Shengli Zhou. 2014. DA-Sync: A Doppler-assisted time-synchronization scheme for mobile underwater sensor networks. IEEE Transactions on Mobile Computing 13, 3, 582-595.

Jun Liu, Zhong Zhou, Zheng Peng, Jun-Hong Cui, Michael Zuba, and Lance Fiondella. 2013. Mobi-Sync: Efficient time synchronization for mobile underwater sensor networks. IEEE Transactions on Parallel and Distributed Systems 24, 2, 406-416.

Michael A. Lombardi. 2001. An introduction to frequency calibrations. Reprinted from NIST Frequency Measurement and Analysis Systems: Operator's Manual. NIST, Gaithersburg, MD, 29.

Michael Kevin Maggs, Steven G O'Keefe, and David Victor Thiel. 2012. Consensus clock synchronization for wireless sensor networks. IEEE Sensors Journal 12, 6, 2269-2277.

Miklós Maróti, Branislav Kusy, Gyula Simon, and Ákos Lédeczi. 2004. The flooding time synchronization protocol. In Proceedings of the Sensys Conference. 39-49.

Microchip. 2008. Run-Time Calibration of Watch Crystals. Available at http://www.microchip.com.

D. L. Mills. 1991. Internet time synchronization: The network time protocol. IEEE Transactions on Communications 39, 10, 1482-1493. DOI : http://dx.doi.org/10.1109/26.103043

National Instruments. 2012. NI USB-6008/6009 User Guide and Specifications. Retrieved Marcy 15, 2016, from http://www.ni.com/pdf/manuals/371303n.pdf.

Su Ping. 2003. Delay Measurement Time Synchronization for Wireless Sensor Networks. Technical Report IRB-TR-03-013. Intel Research.

Joseph Polastre, Jason Hill, and David Culler. 2004. Versatile low power media access for wireless sensor networks. In Proceedings of the SenSys Conference. DOI : http://dx.doi.org/10.1145/1031495.1031508

J. Polastre, R. Szewczyk, and D. Culler. 2005. Telos: Enabling ultra-low power wireless research. In Proceedings of the IPSN Conference. 364-369.

Luca Schenato and Federico Fiorentin. 2011. Average timesynch: A consensus-based protocol for clock synchronization in wireless sensor networks. Automatica 47, 9, 1878-1886.

T. Schmid, Z. Charbiwala, R. Shea, and M. B. Srivastava. 2009. Temperature compensated time synchronization. IEEE Embeded Systems Letters 1, 2. DOI : http://dx.doi.org/10.1109/LES.2009.2028103

Roger S. Strout. 1928. The temperature coefficient of quartz crystal oscillators. Physical Review 32, $5,829$. DOI : http://dx.doi.org/10.1103/PhysRev.32.829

Bharath Sundararaman, Ugo Buy, and Ajay D. Kshemkalyani. 2005. Clock synchronization for wireless sensor networks: A survey. Ad Hoc Networks 3, 281-323.

R. Szewczyk, A. Mainwaring, J. Polastre, J. Anderson, and D. Culler. 2004. An analysis of a large scale habitat monitoring application. In Proceedings of the SenSys Conference. 214-226.

Gilman Tolle, Joseph Polastre, Robert Szewczyk, David Culler, Neil Turner, Kevin Tu, Stephen Burgess, Todd Dawson, Phil Buonadonna, David Gay, and Wei Hong. 2005. A macroscope in the redwoods. In Proceedings of the SenSys Conference. 51-63.

I. Vasilescu, K. Kotay, D. Rus, M. Dunbabin, and P. Corke. 2005. Data collection, storage, and retrieval with an underwater sensor network. In Proceedings of the SenSys Conference. 154-165.

F. L. Walls and J.-J. Gagnepain. 1992. Environmental sensitivities of quartz oscillators. IEEE Transactions on Ultrasonics, Ferroelectrics, and Frequency Control 39, 2, 241-249.

Yik-Chung Wu, Qasim Chaudhari, and Erchin Serpedin. 2011. Clock synchronization of wireless sensor networks. IEEE Signal Processing Magazine 28, 1, 124-138.

Yik-Chung Wu, Long-Fung Cheung, King-Shan Lui, and Philip W. T. Pong. 2012. Efficient communication of sensors monitoring overhead transmission lines. IEEE Transactions on Smart Grid 3, 3, 1130-1136.

Zhe Yang, Lin Cai, Yu Liu, and Jianping Pan. 2012. Environment-aware clock skew estimation and synchronization for wireless sensor networks. In Proceedings of the INFOCOM Conference. 1017-1025. DOI : http://dx.doi.org/10.1109/INFCOM.2012.6195457

Kasim Sinan Yildirim and Aylin Kantarci. 2014. Time synchronization based on slow-flooding in wireless sensor networks. IEEE Transactions on Parallel and Distributed Systems 25, 1, 244-253.

Ziguo Zhong, Pengpeng Chen, and Tian He. 2011. On-demand time synchronization with predictable accuracy. In Proceedings of the INFOCOM Conference. 2480-2488. DOI:http://dx.doi.org/10.1109/ INFCOM.2011.5935071

Received January 2015; revised November 2015; accepted January 2016 